\title{
First observation of the decay $B_{s}^{0} \rightarrow \phi \bar{K}^{* 0}$
}

\section{LHCh}

\section{The LHCb collaboration}

E-mail: antonio.romero@usc.es

Abstract: The first observation of the decay $B_{s}^{0} \rightarrow \phi \bar{K}^{* 0}$ is reported. The analysis is based on a data sample corresponding to an integrated luminosity of $1.0 \mathrm{fb}^{-1}$ of $p p$ collisions at $\sqrt{s}=7 \mathrm{TeV}$, collected with the LHCb detector. A yield of $30 \pm 6 \quad B_{s}^{0} \rightarrow\left(K^{+} K^{-}\right)\left(K^{-} \pi^{+}\right)$decays is found in the mass windows $1012.5<M\left(K^{+} K^{-}\right)<1026.5 \mathrm{MeV} / c^{2}$ and $746<M\left(K^{-} \pi^{+}\right)<1046 \mathrm{MeV} / c^{2}$. The signal yield is found to be dominated by $B_{s}^{0} \rightarrow \phi \bar{K}^{* 0}$ decays, and the corresponding branching fraction is measured to be $\mathcal{B}\left(B_{s}^{0} \rightarrow \phi \bar{K}^{* 0}\right)$ $=\left(1.10 \pm 0.24\right.$ (stat) \pm 0.14 (syst) $\left.\pm 0.08\left(f_{d} / f_{s}\right)\right) \times 10^{-6}$, where the uncertainties are statistical, systematic and from the ratio of fragmentation fractions $f_{d} / f_{s}$ which accounts for the different production rate of $B^{0}$ and $B_{s}^{0}$ mesons. The significance of $B_{s}^{0} \rightarrow \phi \bar{K}^{* 0}$ signal is 6.1 standard deviations. The fraction of longitudinal polarization in $B_{s}^{0} \rightarrow \phi \bar{K}^{* 0}$ decays is found to be $f_{0}=0.51 \pm 0.15$ (stat) \pm 0.07 (syst).

Keywords: B physics, Flavour Changing Neutral Currents, Flavor physics, HadronHadron Scattering

ArXiv EPRINT: 1306.2239 


\section{Contents}

1 Introduction 1

2 Detector and software 3

3 Signal selection $\quad 4$

4 Suppression of background from other b-hadron decays 5

5 Fit to the four-body mass spectrum $\quad 6$

$\begin{array}{lll}6 & \text { Determination of the S-wave contribution } & 7\end{array}$

7 Determination of the $B_{s}^{0} \rightarrow \phi \bar{K}^{* 0}$ branching fraction 8

8 Systematic uncertainties on the branching fraction 10

$9 \begin{array}{ll}9 \text { Polarization analysis } & 10\end{array}$

$\begin{array}{ll}10 \text { Summary and conclusions } & 13\end{array}$

$\begin{array}{lr}\text { The LHCb collaboration } & 18\end{array}$

\section{Introduction}

The measurement of $C P$ asymmetries in flavour-changing neutral-current processes provides a crucial test of the Standard Model (SM). In particular, loop-mediated (penguin) decays of $B$ mesons are sensitive probes for physics beyond the SM. Transitions between the quarks of the third and second generation $(b \rightarrow s)$ or between the quarks of the third and first generation $(b \rightarrow d)$ are complementary since SM $C P$ violation is tiny in $b \rightarrow s$ transitions and an observation of $C P$ violation would indicate physics beyond the SM. For $b \rightarrow d$ transitions the SM branching fraction is an order of magnitude smaller than $b \rightarrow s$ due to the relative suppression of $\left|V_{t d}\right|^{2} /\left|V_{t s}\right|^{2}$. It is particularly useful to have experimental information on pairs of channels related by $d \leftrightarrow s$ exchange symmetry to test that the QCD contribution to the decay is independent of the initial $B^{0}$ or $B_{s}^{0}$ meson.

The BaBar and Belle experiments have performed measurements of $b \rightarrow s q \bar{q}$ processes, such as $B^{0} \rightarrow \phi K_{\mathrm{S}}^{0}, B^{0} \rightarrow \eta^{\prime} K_{\mathrm{S}}^{0}$ and $B^{0} \rightarrow f_{0} K_{\mathrm{S}}^{0}$ [1-3], and of $b \rightarrow d q \bar{q}$ penguin diagrams, such as $B^{0} \rightarrow K_{\mathrm{S}}^{0} K_{\mathrm{S}}^{0}$ and $B^{+} \rightarrow K^{+} K_{\mathrm{S}}^{0}[4,5]$. These modes contain pseudo-scalar or scalar mesons in their final state whereas $B_{(s)}^{0} \rightarrow V V^{\prime}$ decays, where $V$ and $V^{\prime}$ are light vector mesons, provide a valuable additional source of information because the angular distributions give insight into the physics of hadronic $B$ meson decays and the interplay 
between the strong and weak interactions they involve. From the V-A structure of the weak interaction and helicity conservation in the strong interaction, the final state of these decays is expected to be highly longitudinally polarized. This applies to both tree and penguin decays. The BaBar and Belle experiments have confirmed that longitudinal polarization dominates in $b \rightarrow u$ tree processes such as $B^{0} \rightarrow \rho^{+} \rho^{-}[6,7], B^{+} \rightarrow \rho^{0} \rho^{+}[8,9]$ and $B^{+} \rightarrow \omega \rho^{+}$[10]. However, measurements of the polarization in decays with both tree and penguin contributions, such as $B^{0} \rightarrow \rho^{0} K^{* 0}$ and $B^{0} \rightarrow \rho^{-} K^{*+}$ [11] and in $b \rightarrow s$ penguin decays, $B^{0} \rightarrow \phi K^{* 0}[12,13], B_{s}^{0} \rightarrow K^{* 0} \bar{K}^{* 0}[14]$ and $B_{s}^{0} \rightarrow \phi \phi[15-17]$, indicate a low value of the longitudinal polarization fraction comparable with, or even smaller than, the transverse fraction.

The $B_{(s)}^{0} \rightarrow V V^{\prime}$ decays can be described by models based on perturbative QCD, or QCD factorization and $\mathrm{SU}(3)$ flavour symmetries. Whilst some authors predict a longitudinal polarization fraction $f_{0} \sim 0.9$ for tree-dominated and $\sim 0.75$ for penguin decays [18-20], other studies have proposed different mechanisms such as penguin annihilation [21, 22] and QCD rescattering [23] to accommodate smaller longitudinal polarization fractions $\sim 0.5$, although the predictions have large uncertainties. A review on the topic of polarization in $B$ decays can be found in ref. [24].

There are only two other $B_{(s)}^{0} \rightarrow V V^{\prime}$ penguin modes that correspond to $b \rightarrow d$ loops. The first is the $B^{0} \rightarrow K^{* 0} \bar{K}^{* 0}$ decay. The BaBar collaboration reported the discovery of this channel with $6 \sigma$ significance and a measurement of its branching fraction $\mathcal{B}\left(B^{0} \rightarrow\right.$ $\left.K^{* 0} \bar{K}^{* 0}\right)=\left(1.28_{-0.30}^{+0.35} \pm 0.11\right) \times 10^{-6}[25]$. This is in tension with the results of the Belle collaboration that published an upper limit of $\mathcal{B}\left(B^{0} \rightarrow K^{* 0} \bar{K}^{* 0}\right)<0.8 \times 10^{-6}$ at the $90 \%$ confidence level [26]. The BaBar publication also reported a measurement of the longitudinal polarization $f_{0}=0.80_{-0.13}^{+0.12}$ [25], which is large compared to those from $B^{0} \rightarrow \phi K^{* 0}\left(f_{0}=0.494 \pm 0.036[13]\right), B_{s}^{0} \rightarrow \phi \phi\left(f_{0}=0.365 \pm 0.025[16]\right)$ and $B_{s}^{0} \rightarrow K^{* 0} \bar{K}^{* 0}$ $\left(f_{0}=0.31 \pm 0.13[14]\right)$.

The mode $B_{s}^{0} \rightarrow \phi \bar{K}^{* 0}$ is the other $b \rightarrow d$ penguin decay into vector mesons that has not previously been observed. This decay is closely linked to $B^{0} \rightarrow \phi K^{* 0}$, differing in the spectator quark and the final quark in the loop, as shown in figure $1 .^{1}$ From the aforementioned relation between $b \rightarrow s$ and $b \rightarrow d$ transitions, their relative branching fractions should scale as $\left|V_{t d}\right|^{2} /\left|V_{t s}\right|^{2}$ and their polarization fractions are expected to be very similar. Moreover, since both decays share the same final state, except for charge conjugation, $B^{0} \rightarrow \phi K^{* 0}$ is the ideal normalization channel for the determination of the $B_{s}^{0} \rightarrow \phi \bar{K}^{* 0}$ branching fraction. The $B_{s}^{0} \rightarrow \phi \bar{K}^{* 0}$ decay is also related to $B^{0} \rightarrow K^{* 0} \bar{K}^{* 0}$, since their loop diagrams only differ in the spectator quark ( $s$ instead of $d$ ), although it has been suggested that $\mathrm{S}$-wave interference effects might break the $\mathrm{SU}(3)$ symmetry relating two channels [27]. Finally, it is also interesting to explore the relation of the $B_{s}^{0} \rightarrow \phi \bar{K}^{* 0}$ decay with the $B^{0} \rightarrow \rho^{0} K^{* 0}$ mode since the penguin loop diagrams of these modes are related by the $d \leftrightarrow s$ exchange. The $B^{0} \rightarrow \rho^{0} K^{* 0}$ decay also has a $b \rightarrow u$ tree diagram, but it is expected that the penguin contribution is dominant, since the branching fraction

\footnotetext{
${ }^{1}$ Both the decays $B_{s}^{0} \rightarrow \phi \bar{K}^{* 0}$ and $B^{0} \rightarrow \phi K^{* 0}$ could also have contributions from QCD singlet-penguin amplitudes [21].
} 

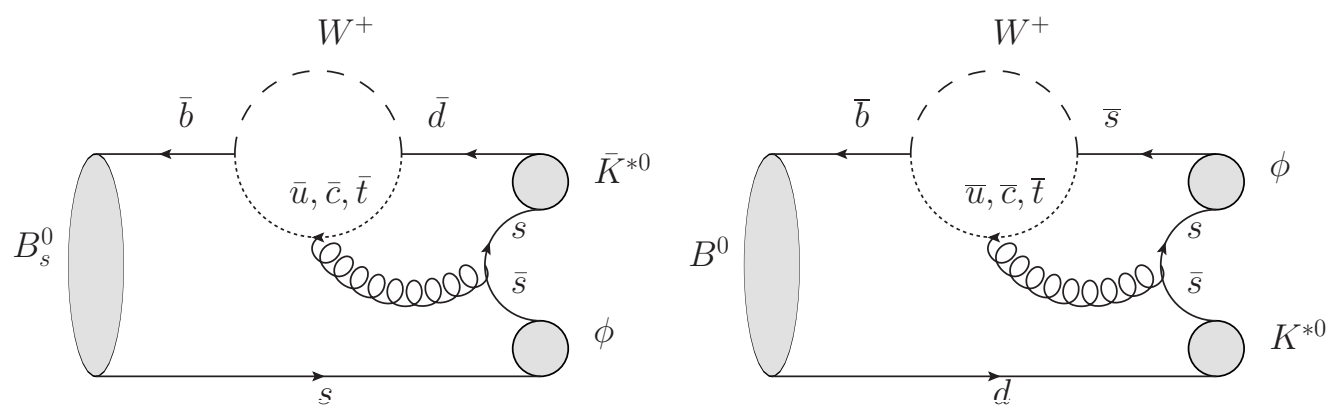

Figure 1. Feynman diagrams for the $B_{s}^{0} \rightarrow \phi \bar{K}^{* 0}$ and the $B^{0} \rightarrow \phi K^{* 0}$ decays.

is comparable to that of the pure penguin $B^{0} \rightarrow \phi K^{* 0}$ decay.

The most stringent previous experimental limit on the $B_{s}^{0} \rightarrow \phi \bar{K}^{* 0}$ branching fraction is $\mathcal{B}\left(B_{s}^{0} \rightarrow \phi \bar{K}^{* 0}\right)<1.0 \times 10^{-3}$ at the $90 \%$ confidence level [24], whereas calculations based on the QCD factorization framework predict a value of $\left(0.4_{-0.3}^{+0.5}\right) \times 10^{-6}$ [21] while in perturbative QCD a value of $\left(0.65_{-0.23}^{+0.33}\right) \times 10^{-6}[28]$ is obtained. The precise determination of the branching fraction tests these models and provides a probe for physics beyond the SM.

The study of the angular distributions in the $B_{s}^{0} \rightarrow \phi \bar{K}^{* 0}$ channel provides a measurement of its polarization. In ref. [28], a prediction of $f_{0}=0.712_{-0.048}^{+0.042}$ is made for the longitudinal polarization fraction, using the perturbative QCD approach, that can be compared to the experimental result.

In this paper the first observation of the $B_{s}^{0} \rightarrow \phi \bar{K}^{* 0}$ decay, with $\phi \rightarrow K^{+} K^{-}$and $\bar{K}^{* 0} \rightarrow K^{-} \pi^{+}$, is reported and the determination of its branching fraction and polarizations are presented. The study is based on data collected by the LHCb experiment at CERN from the $\sqrt{s}=7 \mathrm{TeV}$ proton-proton collisions of LHC beams. The dataset corresponds to an integrated luminosity of $1.0 \mathrm{fb}^{-1}$.

\section{Detector and software}

The LHCb detector [29] is a single-arm forward spectrometer covering the pseudorapidity range $2<\eta<5$, designed for the study of particles containing $b$ or $c$ quarks. The detector includes a high precision tracking system consisting of a silicon-strip vertex detector surrounding the $p p$ interaction region, a large-area silicon-strip detector located upstream of a dipole magnet with a bending power of about $4 \mathrm{Tm}$, and three stations of siliconstrip detectors and straw drift tubes placed downstream. The combined tracking system provides a momentum measurement with relative uncertainty that varies from $0.4 \%$ at $5 \mathrm{GeV} / c$ to $0.6 \%$ at $100 \mathrm{GeV} / c$, and impact parameter resolution of $20 \mu \mathrm{m}$ for tracks with high transverse momentum $\left(p_{\mathrm{T}}\right)$. Charged hadrons are identified using two ring-imaging Cherenkov (RICH) detectors [30]. Photon, electron and hadron candidates are identified by a calorimeter system consisting of scintillating-pad and preshower detectors, an electromagnetic calorimeter and a hadronic calorimeter. Muons are identified by a system composed of alternating layers of iron and multiwire proportional chambers [31]. 
The trigger [32] consists of a hardware stage, based on information from the calorimeter and muon systems, followed by a software stage, which applies a full event reconstruction. The software trigger used in this analysis requires a two-, three- or four-track secondary vertex with a high sum of the $p_{\mathrm{T}}$ of the tracks and significant displacement from the primary $p p$ interaction vertices (PVs). At least one track should have $p_{\mathrm{T}}>1.7 \mathrm{GeV} / c$ and impact parameter $\chi^{2}\left(\chi_{\mathrm{IP}}^{2}\right)$ with respect to all primary interactions greater than 16 . The $\chi_{\mathrm{IP}}^{2}$ is defined as the difference between the $\chi^{2}$ of a PV reconstructed with and without the considered track. A multivariate algorithm [33] is used for the identification of secondary vertices consistent with the decay of a $b$ hadron.

In the simulation, $p p$ collisions are generated using PYTHIA 6.4 [34] with a specific LHCb configuration [35]. Decays of hadronic particles are described by EvTGEN [36], in which final state radiation is generated using Рнотоs [37]. The interaction of the generated particles with the detector and its response are implemented using the GEANT4 toolkit [38, 39] as described in ref. [40].

\section{Signal selection}

Signal $B_{s}^{0} \rightarrow \phi \bar{K}^{* 0}$ candidates are formed from $\phi \rightarrow K^{+} K^{-}$and $\bar{K}^{* 0} \rightarrow K^{-} \pi^{+}$decays. $^{2}$ The pairs of charged particles in the $\phi \rightarrow K^{+} K^{-}$and the $\bar{K}^{* 0} \rightarrow K^{-} \pi^{+}$candidates must combine to give invariant masses $1012.5<M\left(K^{+} K^{-}\right)<1026.5 \mathrm{MeV} / c^{2}$ and $746<M\left(K^{-} \pi^{+}\right)<1046 \mathrm{MeV} / c^{2}$, consistent with the known $\phi$ and $\bar{K}^{* 0}$ masses [24]. Each of the four tracks is required to have $p_{\mathrm{T}}>500 \mathrm{MeV} / c$ and $\chi_{\mathrm{IP}}^{2}>9$.

Kaons and pions are distinguished by use of a log-likelihood algorithm that combines information from the RICH detectors and other properties of the event [30]. The final state particles are identified by requiring that the difference in log-likelihoods of the kaon and pion mass hypotheses is $\operatorname{DLL}_{K \pi}>2$ for each kaon candidate and $<0$ for the pion candidate. In addition, the difference in log-likelihoods of the proton and kaon hypotheses, $\operatorname{DLL}_{p K}$, is required to be $<0$ for the kaon from the $\bar{K}^{* 0}$ decay. This suppresses background from $\Lambda_{b}^{0}$ decays. This requirement is not necessary for the kaons from the $\phi$ candidate owing to the narrow $K^{+} K^{-}$invariant mass window.

The $K^{-} \pi^{+}$pair that forms the $\bar{K}^{* 0}$ candidate is required to originate from a common vertex with a $\chi^{2}$ per number of degrees of freedom $\left(\chi^{2} / \mathrm{ndf}\right)<9$, and to have a positive cosine of the angle between its momentum and the reconstructed $B_{(s)}^{0}$ candidate flight direction, calculated with the $B_{(s)}^{0}$ decay vertex and the best matching primary vertex. The $K^{-} \pi^{+}$combination is also required to have $p_{\mathrm{T}}>900 \mathrm{MeV} / c$. The same conditions are imposed on the $\phi$ candidate.

The $B_{(s)}^{0}$ candidates are also required to fulfil some minimal selection criteria: the $\phi$ and $\bar{K}^{* 0}$ candidates must form a vertex with $\chi^{2} /$ ndf $<15$; the distance of closest approach between their trajectories must be less than $0.3 \mathrm{~mm}$; and they must combine to give an invariant mass within $4866<M\left(K^{+} K^{-} K^{-} \pi^{+}\right)<5866 \mathrm{MeV} / c^{2}$.

In addition, a geometrical-likelihood based selection (GL) [41, 42] is implemented using as input variables properties of the $B_{(s)}^{0}$ meson candidate. These are

\footnotetext{
${ }^{2}$ Inclusion of charge conjugated processes is implied in this work, unless otherwise stated.
} 
- the $B_{(s)}^{0}$ candidate impact parameter (IP) with respect to the closest primary vertex;

- the decay time of the $B_{(s)}^{0}$ candidate;

- the $p_{\mathrm{T}}$ of the $B_{(s)}^{0}$ candidate;

- the minimum $\chi_{\mathrm{IP}}^{2}$ of the four tracks with respect to all primary vertices in the event; and

- the distance of closest approach between the $\bar{K}^{* 0}$ and $\phi$ candidates' trajectories reconstructed from their respective daughter tracks.

The GL is trained to optimize its discrimination power using representative signal and background samples. For the signal a set of $B_{s}^{0} \rightarrow \phi \bar{K}^{* 0}$ simulated events is used. For the background a sample of events where, in addition to the signal selections, other than those on the masses, requirements of $999.5<$ $M\left(K^{+} K^{-}\right)<1012.5 \mathrm{MeV} / c^{2}$ or $1026.5<M\left(K^{+} K^{-}\right)<1039.5 \mathrm{MeV} / c^{2}$ for the $\phi$ candidate and $M\left(K^{+} K^{-} K^{-} \pi^{+}\right)>5413 \mathrm{MeV} / c^{2}$ for the four-body mass are applied. The selection of only the high-mass $B_{(s)}^{0}$ sideband is motivated by the nature of the background in that region, which is purely combinatorial, whereas the low-mass sideband contains partially reconstructed $B$ meson decays that have topological similarities to the signal.

\section{Suppression of background from other $b$-hadron decays}

A small background from $B_{s}^{0} \rightarrow \phi \phi$ decays, where one of the kaons from the $\phi$ is misidentified as a pion, is found to contaminate the signal. Candidate $B_{s}^{0} \rightarrow \phi \bar{K}^{* 0}$ decays are therefore required to be outside of the window defined by $1012.5<M\left(K^{+} K^{-}\right)<1026.5 \mathrm{MeV} / c^{2}$ and $5324<M\left(K^{+} K^{-} K^{+} K^{-}\right)<5424 \mathrm{MeV} / c^{2}$ in the $K^{+} K^{-}$and $K^{+} K^{-} K^{+} K^{-}$invariant masses when the mass hypothesis for the sole pion of the decay is switched into a kaon. In simulated events this selection removes $0.12 \%$ of the $B_{s}^{0} \rightarrow \phi \bar{K}^{* 0}$ signal decays and does not affect the $B^{0} \rightarrow \phi K^{* 0}$ decay mode. Other possible reflections, such as $B_{s}^{0} \rightarrow K^{* 0} \bar{K}^{* 0}$ decays, are found to be negligible.

In order to remove background from $B_{s}^{0} \rightarrow D_{s}^{\mp}\left(\phi \pi^{\mp}\right) K^{ \pm}$decays when the $\pi^{\mp}$ and the $K^{ \pm}$mesons form a $\stackrel{(-)}{K^{* 0}}$ candidate, events with the invariant mass of the $K^{+} K^{-} \pi^{\mp}$ system within $1953.5<M\left(K^{+} K^{-} \pi^{\mp}\right)<1983.5 \mathrm{MeV} / c^{2}$, consistent with the known $D_{s}^{+}$mass [24], are excluded.

Background from $b$-hadron decays containing a misidentified proton has also been considered. For candidate $B_{s}^{0} \rightarrow \phi \bar{K}^{* 0}$ decays, the kaon with the largest $\mathrm{DLL}_{p K}$ is assigned the proton mass and the four-body invariant mass recomputed. The largest potential background contribution arises from $\bar{\Lambda}_{b}^{0} \rightarrow K^{+} K^{-} \bar{p} \pi^{+}$where the antiproton is misidentified as the kaon originating from the $\bar{K}^{* 0}$ meson, and $\Lambda_{b}^{0} \rightarrow K^{+} K^{-} K^{-} p$, where the proton is misidentified as the pion originating from the $\bar{K}^{* 0}$ meson. Simulation shows that these decays produce wide four-body mass distributions which peak around $5450 \mathrm{MeV} / c^{2}$ and $5500 \mathrm{MeV} / c^{2}$, respectively. This background contribution is considered in the fit model discussed below. Other $B_{(s)}^{0}$ decay modes containing a $\Lambda \rightarrow p \pi^{-}$decay or background from $\Lambda_{c}^{+} \rightarrow p K^{-} \pi^{+}$decays are found to be negligible. 


\section{$5 \quad$ Fit to the four-body mass spectrum}

The sample of 1277 candidates, selected as described in sections 3 and 4, contains many $B^{0} \rightarrow \phi K^{* 0}$ decays whereas only a small contribution from $B_{s}^{0} \rightarrow \phi \bar{K}^{* 0}$ decays is anticipated. Both signals are parametrized with identical shapes, differing only in the mass shift of $87.13 \mathrm{MeV} / c^{2}$ between the $B^{0}$ and $B_{s}^{0}$ mesons [24] which is fixed in the fit. The signal shapes are described by the sum of Crystal Ball (CB) [43] and Gaussian functions that share a common mean. The CB function, which contains most of the signal, is a combination of a Gaussian function with a power law tail, accounting for the intrinsic detector resolution and the radiative tail toward low masses, respectively. The Gaussian shape describes events reconstructed with worse mass resolution, which produce a contamination of $B^{0} \rightarrow \phi K^{* 0}$ decays in the region of the $B_{s}^{0} \rightarrow \phi \bar{K}^{* 0}$ signal peak. The dependence between the Gaussian and $\mathrm{CB}$ resolutions, $\sigma_{\mathrm{G}}$ and $\sigma_{\mathrm{CB}}$, respectively, is found to be

$$
\sigma_{\mathrm{G}}=\sqrt{\sigma_{\mathrm{CB}}^{2}+\left(24.74 \mathrm{MeV} / c^{2}\right)^{2}}
$$

from a data sample of $25 \times 10^{3} B^{0} \rightarrow J / \psi K^{* 0}$ decays. This channel is topologically very similar to the signal and is almost background free. The fit to this sample also provides the power law exponent of the $\mathrm{CB}$ function tail, which is subsequently fixed in the $B_{s}^{0} \rightarrow\left(K^{+} K^{-}\right)\left(K^{-} \pi^{+}\right)$and $B^{0} \rightarrow\left(K^{+} K^{-}\right)\left(K^{+} \pi^{-}\right)$mass models. The parameter that governs the transition from the Gaussian shape to the power law function in the $\mathrm{CB}$ function is unrestrained in the fit. The other unrestrained fit parameters include: the central $B$ meson mass, the width of the CB function, the fractional yield contained in the Gaussian function and the total signal yield.

In addition to the $B^{0}$ and $B_{s}^{0}$ signal shapes, three more components are included. The first accounts for partially reconstructed $B$ meson decays into $\phi$ and $K$ or $K^{*}$ excited states where a pion has been lost. This is described by a convolution of the ARGUS shape [44] with a Gaussian distribution. The second contribution is due to $\Lambda_{b}^{0} \rightarrow K^{+} K^{-} K^{-} p$ and $\bar{\Lambda}_{b}^{0} \rightarrow$ $K^{+} K^{-} \bar{p} \pi^{+}$decays and is modelled with a histogram obtained from simplified simulations. The third contribution is an exponential function to account for combinatorial background.

The data passing the selection criteria are fitted using an extended unbinned maximum likelihood fit. The invariant mass distribution of the candidates, together with the fit contribution, is shown in figure 2. The yields of $B_{s}^{0} \rightarrow\left(K^{+} K^{-}\right)\left(K^{-} \pi^{+}\right)$and $B^{0} \rightarrow\left(K^{+} K^{-}\right)\left(K^{+} \pi^{-}\right)$decays are $30 \pm 6$ and $1000 \pm 32$, respectively. The fit model is validated with 10,000 pseudo-experiments, generated with simplified simulations, which show that the signal yields are unbiased. Table 1 summarizes the signal and background contributions resulting from the fit. A likelihood ratio test is employed to assess the statistical significance of the $B_{s}^{0} \rightarrow\left(K^{+} K^{-}\right)\left(K^{-} \pi^{+}\right)$signal yield. This is performed using $\sqrt{2 \ln \left(\mathcal{L}_{\mathrm{s}+\mathrm{b}} / \mathcal{L}_{\mathrm{b}}\right)}$, where $\mathcal{L}_{\mathrm{s}+\mathrm{b}}$ and $\mathcal{L}_{\mathrm{b}}$ are the maximum values of the likelihoods for the signal-plus-background and background-only hypotheses, respectively. ${ }^{3}$ This calculation results in $6.3 \sigma$ significance for the $B_{s}^{0} \rightarrow\left(K^{+} K^{-}\right)\left(K^{-} \pi^{+}\right)$signal. The fit gives

\footnotetext{
${ }^{3}$ The applicability of this method has been verified from the parabolic behaviour of the $B_{s}^{0} \rightarrow\left(K^{+} K^{-}\right)\left(K^{-} \pi^{+}\right)$signal yield profile of $-2 \ln \mathcal{L}_{\mathrm{s}+\mathrm{b}}$ about its minimum.
} 


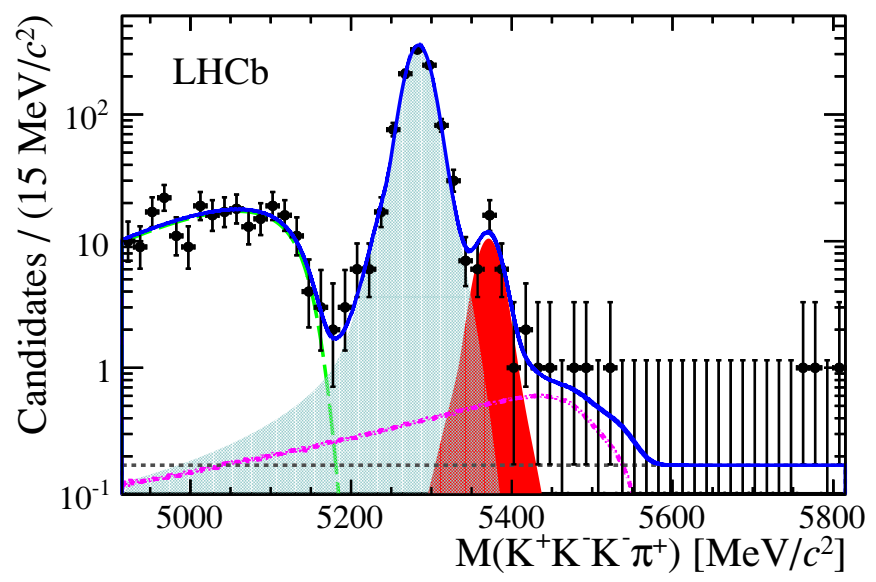

Figure 2. Four-body $K^{+} K^{-} K^{-} \pi^{+}$invariant mass distribution. The points show the data, the blue solid line shows the overall fit, the solid dark red shaded region is the $B_{s}^{0} \rightarrow \phi \bar{K}^{* 0}$ signal, the light blue shaded region corresponds to the $B^{0} \rightarrow \phi K^{* 0}$ signal, the grey dotted line is the combinatorial background and the green dashed line and magenta dashed-dotted lines are the partially reconstructed and misidentified $\Lambda_{b}^{0}$ backgrounds.

$\sigma_{\mathrm{CB}}=15.0 \pm 1.1 \mathrm{MeV} / c^{2}$ for the invariant mass resolution. Integration in a $\pm 30 \mathrm{MeV} / \mathrm{c}^{2}$ mass window yields $26.4 \pm 5.7$ signal candidates and $8.2 \pm 1.3$ background events, composed of $5.4 \pm 0.2$ from $B^{0} \rightarrow\left(K^{+} K^{-}\right)\left(K^{+} \pi^{-}\right), 2.1 \pm 1.3$ from $\Lambda_{b}^{0}$ and $0.7 \pm 0.4$ from combinatorial contributions.

In order to explore systematic effects in the signal yield originating in the fit model two effects were considered. First, the amount of $B^{0} \rightarrow\left(K^{+} K^{-}\right)\left(K^{+} \pi^{-}\right)$events under the $B_{s}^{0} \rightarrow\left(K^{+} K^{-}\right)\left(K^{-} \pi^{+}\right)$signal is governed by the $24.74 \mathrm{MeV} / c^{2}$ factor in eq. (5.1). Similarly, the contamination of misidentified $\Lambda_{b}^{0}$ decays under the signal is controlled by a tail that is parametrized. An extended likelihood is built by multiplying the original likelihood function by Gaussian distributions of these two nuissance parameters with standard deviations of $20 \%$ of their nominal values at which they are centered. The corresponding systematic uncertainty in the signal yield is obtained by performing a fit that maximizes this modified likelihood. The systematic contribution is calculated subtracting the statistical uncertainty in quadrature and found to be \pm 1.2 events. Including this uncertainty results in a significance of $6.2 \sigma$. Effects of other systematic uncertainties, discussed in section 9 , have negiglible impact in the signal significance.

\section{Determination of the S-wave contribution}

The $B_{s}^{0} \rightarrow\left(K^{+} K^{-}\right)\left(K^{-} \pi^{+}\right)$signal is expected to be mainly due to $B_{s}^{0} \rightarrow \phi \bar{K}^{* 0}$ decays, although there are possible non-resonant contributions and $K^{+} K^{-}$and $K^{-} \pi^{+}$pairs from other resonances. To estimate the S-wave contributions, it is assumed that the effect is the same for $B^{0} \rightarrow \phi K^{* 0}$ and $B_{s}^{0} \rightarrow \phi \bar{K}^{* 0}$ decays, therefore allowing the larger sample of $B^{0} \rightarrow \phi K^{* 0}$ decays to be used. The effect of this assumption is considered as a source of systematic uncertainty in section 8 . 


\begin{tabular}{|cc|}
\hline Contribution & Yield \\
\hline$B_{s}^{0} \rightarrow \phi \bar{K}^{* 0}$ & $30 \pm 6$ \\
$B^{0} \rightarrow \phi K^{* 0}$ & $1000 \pm 32$ \\
Partially reconstructed background & $218 \pm 15$ \\
$\Lambda_{b}^{0}$ background & $13 \pm 8$ \\
Combinatorial background & $10 \pm 6$ \\
\hline
\end{tabular}

Table 1. Results of the fit to the sample of selected candidates.

The $K^{+} K^{-}$invariant mass distribution for $\phi$ candidates within a $\pm 30 \mathrm{MeV} / c^{2}$ window of the known $B^{0}$ mass is described by a relativistic spin-1 Breit-Wigner distribution convolved with a Gaussian shape to account for the effect of resolution. A linear term is added to describe the S-wave contribution. The purity resulting from this fit is $0.95 \pm 0.01$ in a $\pm 7 \mathrm{MeV} / c^{2}$ window around the known $\phi$ mass.

The $K^{+} \pi^{-}$pairs are parametrized by the incoherent sum of a relativistic spin-1 BreitWigner amplitude and a shape that describes non-resonant and $K^{* 0}(1430)$ S-wave contributions introduced by the LASS experiment $[13,45]$. The fraction of events from $K^{* 0}$ decays within a $\pm 150 \mathrm{MeV} / c^{2}$ window around the $K^{* 0}$ mass results in a purity of $0.89 \pm 0.02$. When combining the $K^{+} K^{-}$and $K^{+} \pi^{-}$contributions, the total $\phi K^{* 0}$ purity is found to be $0.84 \pm 0.02$. This purity can be translated into a p-value, quantifying the probability that the entire $B_{s}^{0} \rightarrow\left(K^{+} K^{-}\right)\left(K^{-} \pi^{+}\right)$signal is due to decays other than $\phi \bar{K}^{* 0}$. After combining with the $B_{s}^{0} \rightarrow\left(K^{+} K^{-}\right)\left(K^{-} \pi^{+}\right)$significance the $B_{s}^{0} \rightarrow \phi \bar{K}^{* 0}$ is observed with $6.1 \sigma$ significance.

\section{Determination of the $B_{s}^{0} \rightarrow \phi \bar{K}^{* 0}$ branching fraction}

The branching fraction is calculated with the $B^{0} \rightarrow \phi K^{* 0}$ channel as normalization. Both decays pass the same selection and share almost identical topologies. However, since the two decay channels can have different polarizations, their angular distributions may differ which would cause a difference in their detection efficiencies. A factor

$$
\lambda_{f_{0}}=\frac{\epsilon^{B^{0} \rightarrow \phi K^{* 0}}}{\epsilon^{B_{s}^{0} \rightarrow \phi \bar{K}^{* 0}}}=\frac{1-0.29 f_{0}^{B^{0} \rightarrow \phi K^{* 0}}}{1-0.29 f_{0}^{B_{s}^{0} \rightarrow \phi \bar{K}^{* 0}}}
$$

is calculated, where $\epsilon^{B^{0} \rightarrow \phi K^{* 0}}$ and $\epsilon^{B_{s}^{0} \rightarrow \phi \bar{K}^{* 0}}$ are the efficiencies for the $B^{0} \rightarrow \phi K^{* 0}$ and $B_{s}^{0} \rightarrow \phi \bar{K}^{* 0}$ decays reconstruction, $f_{0}^{B^{0} \rightarrow \phi K^{* 0}}$ and $f_{0}^{B_{s}^{0} \rightarrow \phi \bar{K}^{* 0}}$ their longitudinal polarization fractions, determined in section 9 for the $B_{s}^{0} \rightarrow \phi \bar{K}^{* 0}$ mode, and the factor 0.29 is obtained from simulation.

The value of $\mathcal{B}\left(B_{s}^{0} \rightarrow \phi \bar{K}^{* 0}\right)$ is computed from

$$
\mathcal{B}\left(B_{s}^{0} \rightarrow \phi \bar{K}^{* 0}\right)=\lambda_{f_{0}} \times \frac{f_{d}}{f_{s}} \times \mathcal{B}\left(B^{0} \rightarrow \phi K^{* 0}\right) \times \frac{N_{B_{s}^{0} \rightarrow \phi \bar{K}^{* 0}}}{N_{B^{0} \rightarrow \phi K^{* 0}}}
$$



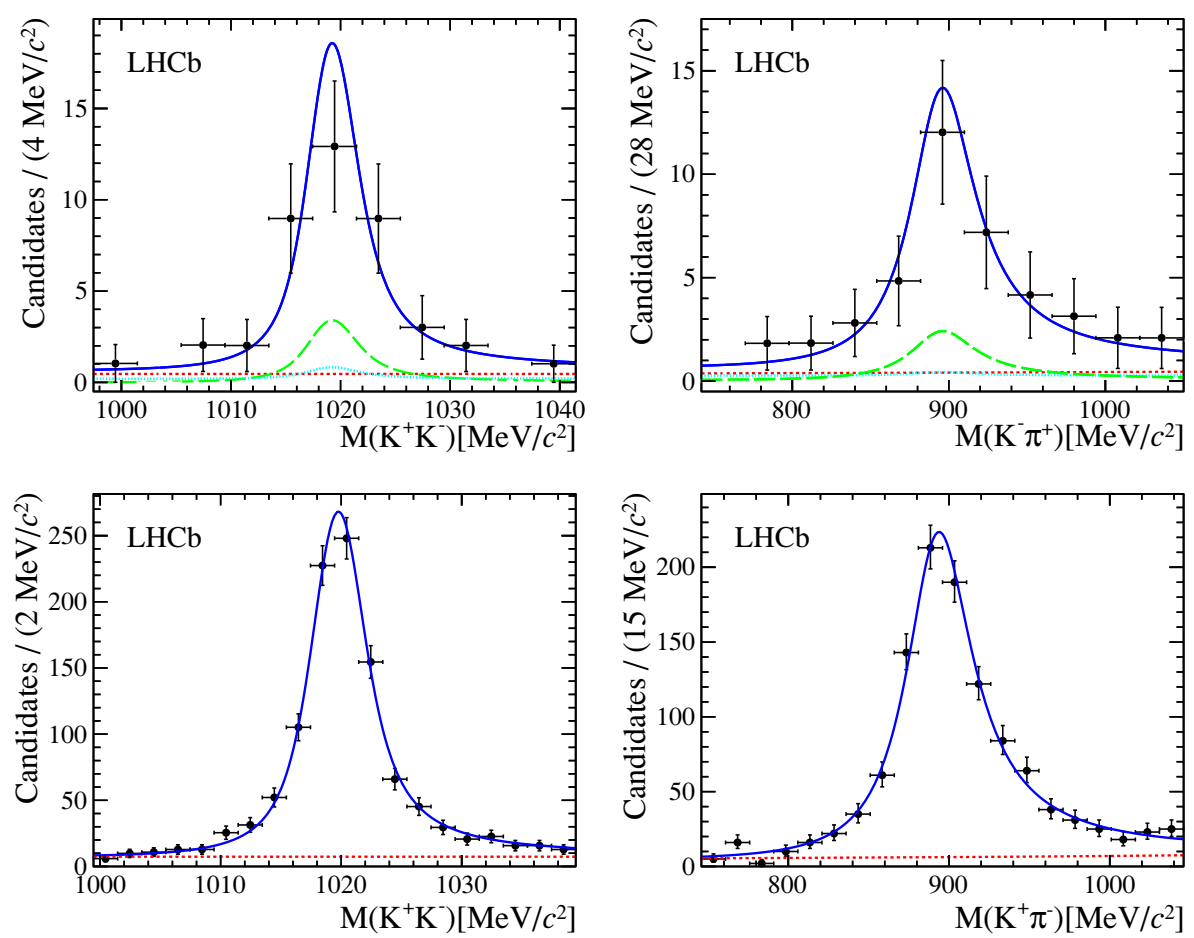

Figure 3. Invariant mass distributions for (left) $K^{+} K^{-}$and (right) $K^{\mp} \pi^{ \pm}$pairs in a $\pm 30 \mathrm{MeV} / c^{2}$ window around the (top) $B_{s}^{0}$ and (bottom) $B^{0}$ mass. The solid blue line is the overall fit, the green dashed line corresponds to $B^{0}$ cross-feed into the $B_{s}^{0}$ mass window, the red dotted line is the S-wave contribution and the light blue is the combinatorial background.

\begin{tabular}{|cc|}
\hline Parameter & Value \\
\hline$\lambda_{f_{0}}$ & $1.01 \pm 0.06$ \\
$N_{B^{0} \rightarrow \phi K^{* 0}}$ & $1000 \pm 32$ \\
$N_{B_{s}^{0} \rightarrow \phi \bar{K}^{* 0}}$ & $30 \pm 6$ \\
$\mathcal{B}\left(B^{0} \rightarrow \phi K^{* 0}\right)$ & $(9.8 \pm 0.6) \times 10^{-6}[24]$ \\
\hline
\end{tabular}

Table 2. Input values for the branching fraction computation.

where $N_{B_{s}^{0} \rightarrow \phi \bar{K}^{* 0}}$ and $N_{B^{0} \rightarrow \phi K^{* 0}}$ are the numbers of $B_{s}^{0}$ and $B^{0}$ decays, respectively, and $f_{d} / f_{s}=3.75 \pm 0.29[46]$ is the ratio of hadronization factors needed to account for the different production rates of $B^{0}$ and $B_{s}^{0}$ mesons. With the values given in table 2 , the result,

$$
\mathcal{B}\left(B_{s}^{0} \rightarrow \phi \bar{K}^{* 0}\right)=(1.10 \pm 0.24) \times 10^{-6},
$$

is obtained, where only the statistical uncertainty is shown.

As a cross-check, a different decay mode, $B^{0} \rightarrow J / \psi K^{* 0}$, with $J / \psi \rightarrow \mu^{+} \mu^{-}$, has been used as a normalization channel. Special requirements were imposed to harmonize the selection of this reference with that for the signal. The obtained result is fully compatible with the $B^{0} \rightarrow \phi K^{* 0}$ based value. 


\section{Systematic uncertainties on the branching fraction}

Four main sources of systematic effects in the determination of the branching fraction are identified: the fit model, the dependence of the acceptance on the longitudinal polarization, the purity of the signal and the uncertainty in the relative efficiency of $B_{s}^{0}$ and $B^{0}$ detection.

Alternatives to the fit model discussed in section 5 give an uncertainty of \pm 1.2 in the signal yield. This results in a relative systematic uncertainty of \pm 0.04 on the branching fraction.

The systematic uncertainty in the acceptance correction factor $\lambda_{f_{0}}$ originates from the uncertainties of the longitudinal polarization fractions, $f_{0}$, in the $B_{s}^{0} \rightarrow \phi \bar{K}^{* 0}$ and $B^{0} \rightarrow \phi K^{* 0}$ channels and is found to be \pm 0.06 .

As described in section 6 an S-wave contribution of $0.16 \pm 0.02$ was found in the $K^{+} K^{-}$and $K^{-} \pi^{+}$mass windows of the $B^{0} \rightarrow \phi K^{* 0}$ candidates. The uncertainty caused by the assumption that this fraction is the same in $B^{0}$ and $B_{s}^{0}$ decays is estimated to be $50 \%$ of the $\mathrm{S}$-wave contribution. This results in a \pm 0.08 contribution to the systematic uncertainty. This uncertainty also accounts for uncanceled interference terms between the $K^{* 0}$, the $\phi$ and their corresponding S-waves. These contributions are linear in the sine or cosine of polarization angles [13] and cancel after integration. The dependence of the acceptance on the angles violates this cancellation contributing \pm 0.04 to the total \pm 0.08 S-wave uncertainty.

The $B_{s}^{0} \rightarrow \phi \bar{K}^{* 0}$ and $B^{0} \rightarrow \phi K^{* 0}$ final states are very similar and a detector acceptance efficiency ratio $\sim 1$ is expected. However, small effects, such as the mass shift $M\left(B_{s}^{0}\right)-M\left(B^{0}\right)$, translate into slightly different $p_{\mathrm{T}}$ distributions for the daughter particles. This results in an efficiency ratio of 1.005, as determined from simulation. The deviation of \pm 0.005 from unity is taken as a systematic uncertainty that is propagated to the branching fraction.

Finally, the uncertainty in the knowledge of the $B^{0} \rightarrow \phi K^{* 0}$ decay branching fraction of $\pm 0.6 \times 10^{-6}$ is also accounted for and results in a relative uncertainty of 0.06 in the $B_{s}^{0} \rightarrow \phi \bar{K}^{* 0}$ decay branching fraction.

A summary of the systematic uncertainties is shown in table 3 . The final result for the $B_{s}^{0} \rightarrow \phi \bar{K}^{* 0}$ decay branching fraction is

$$
\mathcal{B}\left(B_{s}^{0} \rightarrow \phi \bar{K}^{* 0}\right)=\left(1.10 \pm 0.24 \text { (stat) } \pm 0.14 \text { (syst) } \pm 0.08\left(\frac{f_{d}}{f_{s}}\right)\right) \times 10^{-6}
$$

which corresponds to a ratio with the $B^{0} \rightarrow \phi K^{* 0}$ decay branching fraction of

$$
\frac{\mathcal{B}\left(B_{s}^{0} \rightarrow \phi \bar{K}^{* 0}\right)}{\mathcal{B}\left(B^{0} \rightarrow \phi K^{* 0}\right)}=0.113 \pm 0.024 \text { (stat) } \pm 0.013 \text { (syst) } \pm 0.009\left(\frac{f_{d}}{f_{s}}\right) \text {. }
$$

\section{Polarization analysis}

The $B_{s}^{0} \rightarrow \phi \bar{K}^{* 0} \rightarrow\left(K^{+} K^{-}\right)\left(K^{-} \pi^{+}\right)$decay proceeds via two intermediate spin-1 particles. The angular distribution of the decay is described by three transversity amplitudes $A_{0}, A_{\|}$ 


\begin{tabular}{|cc|}
\hline Source & Relative uncertainty in $\mathcal{B}$ \\
\hline Fit model & 0.04 \\
$f_{0}$ & 0.06 \\
Purity & 0.08 \\
Acceptance & 0.005 \\
$\mathcal{B}\left(B^{0} \rightarrow \phi K^{* 0}\right)$ & 0.06 \\
\hline Total & 0.12 \\
\hline
\end{tabular}

Table 3. Sources of systematic uncertainty in the branching fraction measurement. The total uncertainty is the addition in quadrature of the individual sources.

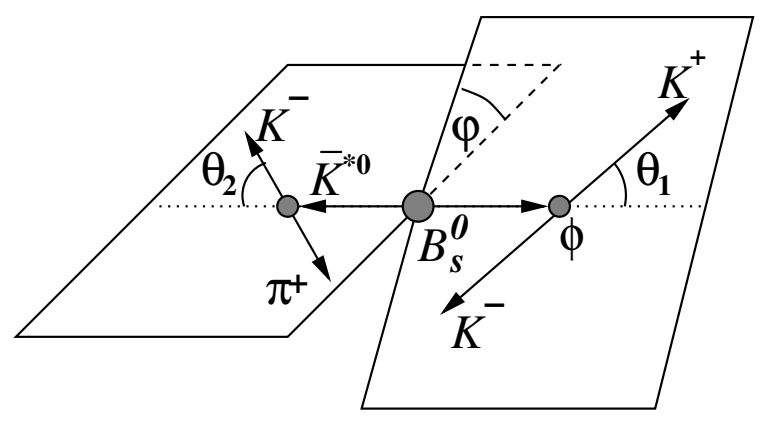

Figure 4. Definition of the angles in $B_{s}^{0} \rightarrow \phi \bar{K}^{* 0}$ decays where $\theta_{1}\left(\theta_{2}\right)$ is the $K^{+}\left(K^{-}\right)$emission angle with respect to the direction opposite to the $B_{s}^{0}$ meson in the $\phi\left(\bar{K}^{* 0}\right)$ rest frame and $\varphi$ is the angle between the $\bar{K}^{* 0}$ and $\phi$ decay planes in the $B_{s}^{0}$ rest frame.

and $A_{\perp}$ [47]. These can be obtained from the distribution of the decay products in three angles $\theta_{1}, \theta_{2}$ and $\varphi$, defined in the helicity frame. The convention for the angles is shown in figure 4. A flavour-averaged and time-integrated polarization analysis is performed assuming that the $C P$-violating phase is zero and that an equal amount of $B_{s}^{0}$ and $\bar{B}_{s}^{0}$ mesons are produced. Under these assumptions, the decay rate dependence on the polarization angles can be written as

$$
\begin{aligned}
\frac{\mathrm{d}^{3} \Gamma}{\mathrm{d} \cos \theta_{1} \mathrm{~d} \cos \theta_{2} \mathrm{~d} \varphi} & \propto\left|A_{0}\right|^{2} \cos ^{2} \theta_{1} \cos ^{2} \theta_{2}+\left|A_{\|}\right|^{2} \frac{1}{2} \sin ^{2} \theta_{1} \sin ^{2} \theta_{2} \cos ^{2} \varphi \\
& +\left|A_{\perp}\right|^{2} \frac{1}{2} \sin ^{2} \theta_{1} \sin ^{2} \theta_{2} \sin ^{2} \varphi+\left|A_{0} \| A_{\|}\right| \cos \delta_{\|} \frac{1}{2 \sqrt{2}} \sin 2 \theta_{1} \sin 2 \theta_{2} \cos \varphi
\end{aligned}
$$

Additional terms accounting for the S-wave and interference contributions, as in ref. [13], are also considered. These terms are set to the values obtained for the $B^{0} \rightarrow \phi K^{* 0}$ sample.

The polarization fractions are defined from the amplitudes as: $f_{j}=\left|A_{j}\right|^{2} /\left(\left|A_{0}\right|^{2}+\right.$ $\left|A_{\|}\right|^{2}+\left|A_{\perp}\right|^{2}$ ) (with $j=0, \|, \perp$ ). In addition to the polarization fractions the cosine of the phase difference between $A_{0}$ and $A_{\|}, \cos \delta_{\|}$, is accessible in this study.

The determination of the angular amplitudes depends on the spectrometer acceptance as a function of the polarization angles $\theta_{1}$ and $\theta_{2}$. The acceptance was found not to depend on $\varphi$. A parametrization of the acceptance as a function of $\theta_{1}$ and $\theta_{2}$ is calculated using 

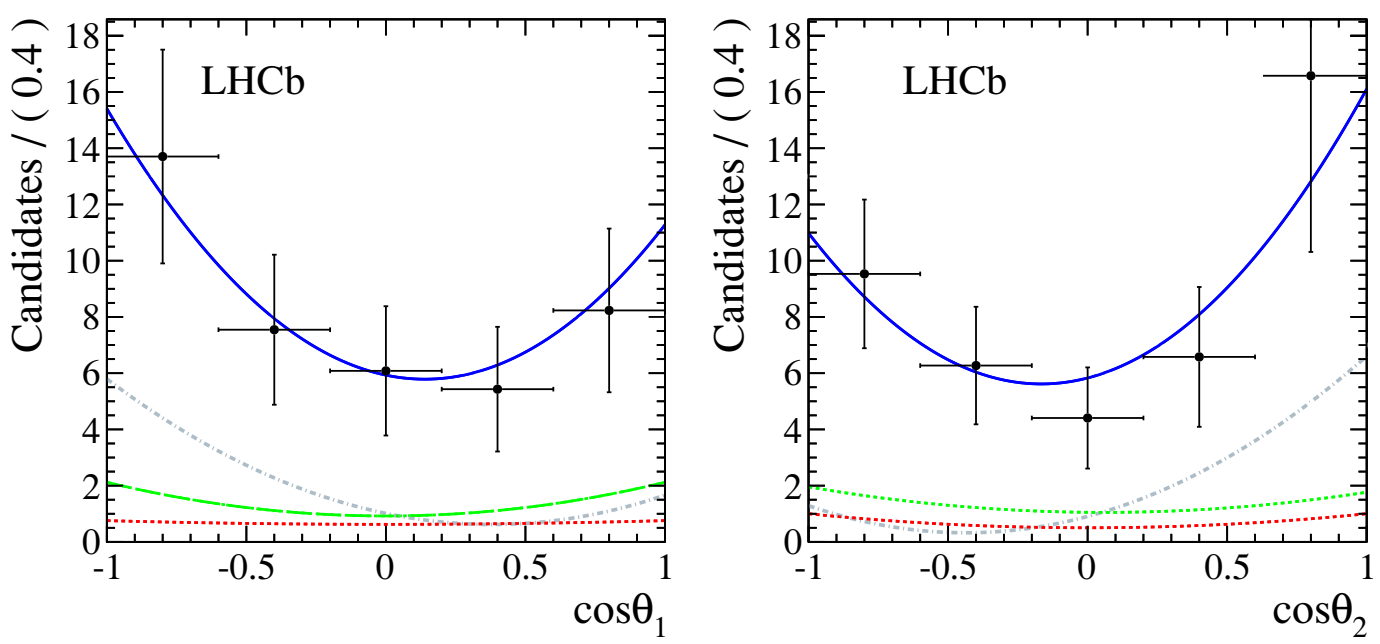

Figure 5. Result of the fit to the angular distribution of the $B_{s}^{0} \rightarrow \phi \bar{K}^{* 0}$ candidates in (left) $\cos \theta_{1}$ and (right) $\cos \theta_{2}$. The red dotted line corresponds to the combinatorial background under the $B_{s}^{0}$ signal, the green dashed line is the $B^{0} \rightarrow \phi K^{* 0}$ signal in the $B_{s}^{0}$ region and the grey dotted-dashed line corresponds to the sum of the S-wave and the interference terms.

simulated data and is used to correct the differential decay rate by scaling eq. (9.1). Additionally, a small correction for discrepancies in the $p_{\mathrm{T}}$ spectrum and the trigger selection of the $B$ mesons between simulation and data is introduced.

The data in a $\pm 30 \mathrm{MeV} / c^{2}$ window around the $B_{s}^{0}$ mass are fitted to the final angular distribution. The fit accounts for two additional ingredients: the tail of the $B^{0} \rightarrow \phi K^{* 0}$ decays, that are polarized with a longitudinal polarization fraction of $f_{0}=0.494$ [13], and the combinatorial background, parametrized from the distributions of events in the high-mass $B$ sideband $5450<M\left(K^{+} K^{-} K^{-} \pi^{+}\right)<5840 \mathrm{MeV} / c^{2}$ after relaxing the selection requirements. The latter accounts for both the combinatorial and misidentified $\Lambda_{b}^{0}$ backgrounds.

The systematic uncertainties in the determination of the angular parameters are calculated modifying the analysis and computing the difference with the nominal result. Three elements are considered.

- The uncertainty in the S-wave fraction. This is computed modifying the S-wave contribution by $50 \%$ of its value. This covers within $2 \sigma$ an S-wave fraction from 0 to $30 \%$, consistent with that typically found in decays of $B$ mesons to final states containing a $K^{* 0}$ meson.

- The spectrometer acceptance. This contribution is calculated comparing the results considering or neglecting the above-mentioned $p_{\mathrm{T}}$ and trigger corrections to the acceptance.

- The combinatorial background. The background model derived from the $B$ mass sideband is replaced by a uniform angular distribution.

The different contributions to the systematic uncertainty are given in table 4 and the one-dimensional projections of the angular distributions are shown figure 5. Other 


\begin{tabular}{|clll|}
\hline Effect & \multicolumn{1}{c}{$\Delta f_{0}$} & $\Delta f_{\|}$ & $\Delta \cos \delta_{\|}$ \\
\hline S-wave & 0.07 & 0.02 & 0.29 \\
Acceptance & 0.007 & 0.005 & 0.002 \\
Combinatorial background & 0.02 & 0.01 & 0.01 \\
\hline Total & 0.07 & 0.02 & 0.29 \\
\hline
\end{tabular}

Table 4. Systematic uncertainties of the angular parameters.

possible systematic sources, such as the uncertainty in the polarization parameters of the $B^{0} \rightarrow \phi K^{* 0}$, are found to be negligible.

Considering all the above, the values obtained are

$$
\begin{aligned}
f_{0} & =0.51 \pm 0.15 \text { (stat) } \pm 0.07 \text { (syst) } \\
f_{\|} & =0.21 \pm 0.11 \text { (stat) } \pm 0.02 \text { (syst) } \\
\cos \delta_{\|} & =-0.18 \pm 0.52(\text { stat }) \pm 0.29 \text { (syst) },
\end{aligned}
$$

and $f_{\perp}=0.28 \pm 0.12$ (stat) \pm 0.03 (syst). These results for the $B_{s}^{0} \rightarrow \phi \bar{K}^{* 0}$ decay are consistent with the values measured in $B^{0} \rightarrow \phi K^{* 0}$ decays of $f_{0}=0.494 \pm 0.036, f_{\perp}=$ $0.212 \pm 0.035$ and $\cos \delta_{\|}=-0.74 \pm 0.10[13]$.

\section{Summary and conclusions}

A total of $30 \pm 6 B_{s}^{0} \rightarrow\left(K^{+} K^{-}\right)\left(K^{-} \pi^{+}\right)$candidates have been observed within the mass windows $1012.5<M\left(K^{+} K^{-}\right)<1026.5 \mathrm{MeV} / c^{2}$ and $746<M\left(K^{-} \pi^{+}\right)<1046 \mathrm{MeV} / c^{2}$. The result translates into a significance of $6.2 \sigma$. The analysis of the $K^{+} K^{-}$and the $K^{-} \pi^{+}$ mass distributions is consistent with $(84 \pm 2) \%$ of the signal originating from resonant $\phi$ and $\bar{K}^{* 0}$ mesons. The significance of the $B_{s}^{0} \rightarrow \phi \bar{K}^{* 0}$ resonant contribution is calculated to be $6.1 \sigma$. The branching fraction of the decay is measured to be

$$
\mathcal{B}\left(B_{s}^{0} \rightarrow \phi \bar{K}^{* 0}\right)=\left(1.10 \pm 0.24(\text { stat }) \pm 0.14 \text { (syst) } \pm 0.08\left(\frac{f_{d}}{f_{s}}\right)\right) \times 10^{-6},
$$

using the $B^{0} \rightarrow \phi K^{* 0}$ decay as a normalization channel. This result is roughly three times the theoretical expectation in QCD factorization of $\left(0.4_{-0.3}^{+0.5}\right) \times 10^{-6}[21]$ and larger than the perturbative QCD value of $\left(0.65_{-0.23}^{+0.33}\right) \times 10^{-6}[28]$, although the values are compatible within $1 \sigma$. The result is also higher than the expectation of $\mathcal{B}\left(B^{0} \rightarrow \phi K^{* 0}\right) \times\left|V_{t d}\right|^{2} /\left|V_{t s}\right|^{2}$. Better precision on both the theoretical and experimental values would allow this channel to serve as a probe for physics beyond the SM.

An angular analysis of the $B_{s}^{0} \rightarrow \phi \bar{K}^{* 0}$ decay results in the polarization fractions and phase difference

$$
\begin{aligned}
f_{0} & =0.51 \pm 0.15(\text { stat }) \pm 0.07 \text { (syst) }, \\
f_{\|} & =0.21 \pm 0.11 \text { (stat) } \pm 0.02(\text { syst }), \\
\cos \delta_{\|} & =-0.18 \pm 0.52(\text { stat }) \pm 0.29 \text { (syst) } .
\end{aligned}
$$


The small value obtained for the longitudinal polarization fraction follows the trend of the $b \rightarrow s$ penguin decays $B^{0} \rightarrow \phi K^{* 0}, B_{s}^{0} \rightarrow K^{* 0} \bar{K}^{* 0}$ and $B_{s}^{0} \rightarrow \phi \phi$. The comparison with the decay $B^{0} \rightarrow K^{* 0} \bar{K}^{* 0}$, where $f_{0}=0.80_{-0.13}^{+0.12} \quad[25]$, shows a $2 \sigma$ discrepancy. This is very interesting since the loop-mediated amplitudes of each decay differ only in the flavour of the spectator quark. The result is also compatible with the longitudinal polarization fraction $f_{0}=0.40 \pm 0.14$ measured in $B^{0} \rightarrow \rho^{0} K^{* 0}$ decays [11], the penguin amplitude of which is related to $B_{s}^{0} \rightarrow \phi \bar{K}^{* 0}$ by $d \leftrightarrow s$ exchange. Finally, the result is smaller than the prediction of perturbative $\mathrm{QCD}, f_{0}=0.712_{-0.048}^{+0.042}$, given in ref. [28].

\section{Acknowledgments}

We express our gratitude to our colleagues in the CERN accelerator departments for the excellent performance of the LHC. We thank the technical and administrative staff at the LHCb institutes. We acknowledge support from CERN and from the national agencies: CAPES, CNPq, FAPERJ and FINEP (Brazil); NSFC (China); CNRS/IN2P3 and Region Auvergne (France); BMBF, DFG, HGF and MPG (Germany); SFI (Ireland); INFN (Italy); FOM and NWO (The Netherlands); SCSR (Poland); ANCS/IFA (Romania); MinES, Rosatom, RFBR and NRC "Kurchatov Institute" (Russia); MinECo, XuntaGal and GENCAT (Spain); SNSF and SER (Switzerland); NAS Ukraine (Ukraine); STFC (United Kingdom); NSF (USA). We also acknowledge the support received from the ERC under FP7. The Tier1 computing centres are supported by IN2P3 (France), KIT and BMBF (Germany), INFN (Italy), NWO and SURF (The Netherlands), PIC (Spain), GridPP (United Kingdom). We are thankful for the computing resources put at our disposal by Yandex LLC (Russia), as well as to the communities behind the multiple open source software packages that we depend on.

Open Access. This article is distributed under the terms of the Creative Commons Attribution License which permits any use, distribution and reproduction in any medium, provided the original author(s) and source are credited.

\section{References}

[1] BeLle collaboration, K. Abe et al., Measurement of time dependent CP-violating asymmetries in $B^{0} \rightarrow \phi K_{s}^{0}, K^{+} K^{-} K_{s}^{0}$ and $\eta^{\prime} K_{s}^{0}$ decays, Phys. Rev. Lett. 91 (2003) 261602 [hep-ex/0308035] [INSPIRE].

[2] BABAR collaboration, B. Aubert et al., Measurements of branching fractions and time-dependent CP-violating asymmetries in $B \rightarrow \eta^{\prime} K$ decays, Phys. Rev. Lett. 94 (2005) 191802 [hep-ex/0502017] [INSPIRE].

[3] BABAR collaboration, B. Aubert et al., Measurement of $C P$ asymmetries in $B^{0} \rightarrow \phi K^{0}$ and $B^{0} \rightarrow K^{+} K^{-} K_{S}^{0}$ decays, Phys. Rev. D 71 (2005) 091102 [hep-ex/0502019] [INSPIRE].

[4] BABAR collaboration, B. Aubert et al., Observation of $B^{+} \rightarrow \bar{K}^{0} K^{+}$and $B^{0} \rightarrow K^{0} \bar{K}^{0}$, Phys. Rev. Lett. 97 (2006) 171805 [hep-ex/0608036] [INSPIRE]. 
[5] Belle collaboration, Y. Nakahama et al., Measurement of time-dependent CP-violating parameters in $B^{0} \rightarrow K_{s}^{0} K_{s}^{0}$ decays, Phys. Rev. Lett. 100 (2008) 121601 [arXiv:0712.4234] [INSPIRE].

[6] Belle collaboration, A. Somov et al., Improved measurement of CP-violating parameters in $B^{0} \rightarrow \rho^{+} \rho^{-}$decays, Phys. Rev. D 76 (2007) 011104 [hep-ex/0702009] [INSPIRE].

[7] Babar collaboration, B. Aubert et al., A study of $B^{0} \rightarrow \rho^{+} \rho^{-}$decays and constraints on the CKM angle $\alpha$, Phys. Rev. D 76 (2007) 052007 [arXiv:0705.2157] [InSPIRE].

[8] Belle collaboration, J. Zhang et al., Observation of $B^{+} \rightarrow \rho^{+} \rho^{0}$, Phys. Rev. Lett. 91 (2003) 221801 [hep-ex/0306007] [INSPIRE].

[9] BABAR collaboration, B. Aubert et al., Improved Measurement of $B^{+} \rightarrow \rho^{+} \rho^{0}$ and determination of the quark-mixing phase angle $\alpha$, Phys. Rev. Lett. 102 (2009) 141802 [arXiv:0901.3522] [INSPIRE].

[10] BABAR collaboration, B. Aubert et al., Observation of $B$ meson decays to $\omega K^{*}$ and improved measurements for $\omega \rho$ and $\omega f_{0}$, Phys. Rev. D 79 (2009) 052005 [arXiv:0901.3703] [INSPIRE].

[11] BABAR collaboration, J.P. Lees et al., $B^{0}$ meson decays to $\rho^{0} K^{* 0}, f_{0} K^{* 0}$ and $\rho^{-} K^{*+}$, including higher $K^{*}$ resonances, Phys. Rev. D 85 (2012) 072005 [arXiv:1112.3896] [INSPIRE].

[12] Belle collaboration, K.-F. Chen et al., Measurement of polarization and triple-product correlations in $B \rightarrow \phi K^{*}$ decays, Phys. Rev. Lett. 94 (2005) 221804 [hep-ex/0503013] [INSPIRE].

[13] BABAR collaboration, B. Aubert et al., Time-dependent and time-integrated angular analysis of $B \rightarrow \phi K_{s} \pi^{0}$ and $B \rightarrow \phi K^{+} \pi^{-}$, Phys. Rev. D 78 (2008) 092008 [arXiv:0808.3586] [INSPIRE].

[14] LHCb collaboration, First observation of the decay $B_{s}^{0} \rightarrow K^{* 0} \bar{K}^{* 0}$, Phys. Lett. B 709 (2012) 50 [arXiv:1111.4183] [INSPIRE].

[15] CDF collaboration, T. Aaltonen et al., Measurement of polarization and search for CP-violation in $B_{s}^{0} \rightarrow \phi \phi$ decays, Phys. Rev. Lett. 107 (2011) 261802 [arXiv:1107.4999] [INSPIRE].

[16] LHCb collaboration, Measurement of the polarization amplitudes and triple product asymmetries in the $B_{s}^{0} \rightarrow \phi \phi$ decay, Phys. Lett. B 713 (2012) 369 [arXiv:1204.2813] [INSPIRE].

[17] LHCb collaboration, First measurement of the CP-violating phase in $B_{s}^{0} \rightarrow \phi \phi$ decays, Phys. Rev. Lett. 110 (2013) 241802 [arXiv:1303.7125] [INSPIRE].

[18] A. Ali, J. Korner, G. Kramer and J. Willrodt, Nonleptonic weak decays of bottom mesons, Z. Phys. C 1 (1979) 269 [INSPIRE].

[19] M. Suzuki, Helicity conservation in inclusive nonleptonic decay $B \rightarrow V X$ : test of long distance final state interaction, Phys. Rev. D 66 (2002) 054018 [hep-ph/0206291] [INSPIRE].

[20] C.-H. Chen, Y.-Y. Keum and H.-n. Li, Perturbative QCD analysis of $B \rightarrow \phi K^{*}$ decays, Phys. Rev. D 66 (2002) 054013 [hep-ph/0204166] [INSPIRE].

[21] M. Beneke, J. Rohrer and D. Yang, Branching fractions, polarisation and asymmetries of $B \rightarrow V V$ decays, Nucl. Phys. B 774 (2007) 64 [hep-ph/0612290] [InSPIRE]. 
[22] H.-Y. Cheng and K.-C. Yang, Branching ratios and polarization in $B \rightarrow V V, V A, A A$ decays, Phys. Rev. D 78 (2008) 094001 [Erratum ibid. D 79 (2009) 039903] [arXiv:0805.0329] [INSPIRE].

[23] H.-Y. Cheng, C.-K. Chua and A. Soni, Final state interactions in hadronic B decays, Phys. Rev. D 71 (2005) 014030 [hep-ph/0409317] [INSPIRE].

[24] Particle Data Group collaboration, J. Beringer et al., Review of particle physics, Phys. Rev. D 86 (2012) 010001 [INSPIRE].

[25] BABAR collaboration, B. Aubert et al., Observation of $B^{0} \rightarrow K^{* 0} \bar{K}^{* 0}$ and search for $B^{0} \rightarrow K^{* 0} K^{* 0}$, Phys. Rev. Lett. 100 (2008) 081801 [arXiv:0708. 2248] [INSPIRE].

[26] Belle collaboration, C.-C. Chiang et al., Search for $B^{0} \rightarrow K^{* 0} \bar{K}^{* 0}, B^{0} \rightarrow K^{* 0} K^{* 0}$ and $B^{0} \rightarrow K^{+} \pi^{-} K^{\mp} \pi^{ \pm}$decays, Phys. Rev. D 81 (2010) 071101 [arXiv: 1001.4595] [InSPIRE].

[27] M. Gronau, O.F. Hernandez, D. London and J.L. Rosner, Electroweak penguins and two-body $B$ decays, Phys. Rev. D 52 (1995) 6374 [hep-ph/9504327] [INSPIRE].

[28] A. Ali et al., Charmless non-leptonic $B_{s}$ decays to $P P, P V$ and $V V$ final states in the $p Q C D$ approach, Phys. Rev. D 76 (2007) 074018 [hep-ph/0703162] [INSPIRE].

[29] LHCb collaboration, The LHCb detector at the LHC, 2008 JINST 3 S08005 [INSPIRE].

[30] M. Adinolfi, et al., Performance of the LHCb RICH detector at the LHC, Eur. Phys. J. C 73 (2013) 2431 [arXiv: 1211.6759] [inSPIRE].

[31] A.A. Alves Jr. et al., Performance of the LHCb muon system, 2013 JINST 8 P02022 [arXiv: 1211.1346] [INSPIRE].

[32] R. Aaij et al., The LHCb trigger and its performance in 2011, 2013 JINST 8 P04022 [arXiv:1211.3055] [INSPIRE].

[33] V.V. Gligorov and M. Williams, Efficient, reliable and fast high-level triggering using a bonsai boosted decision tree, 2013 JINST 8 P02013 [arXiv:1210.6861] [INSPIRE].

[34] T. Sjöstrand, S. Mrenna and P.Z. Skands, PYTHIA 6.4 physics and manual, JHEP 05 (2006) 026 [hep-ph/0603175] [INSPIRE].

[35] I. Belyaev et al., Handling of the generation of primary events in Gauss, the LHCb simulation framework, IEEE Nucl. Sci. Symp. Conf. Rec. (2010) 1155.

[36] D.J. Lange, The EvtGen particle decay simulation package, Nucl. Instrum. Meth. A 462 (2001) 152 [INSPIRE].

[37] P. Golonka and Z. Was, PHOTOS Monte Carlo: a precision tool for QED corrections in Z and $W$ decays, Eur. Phys. J. C 45 (2006) 97 [hep-ph/0506026] [INSPIRE].

[38] GEANT4 collaboration, J. Allison et al., GEANT4 developments and applications, IEEE Trans. Nucl. Sci. 53 (2006) 270.

[39] GEANT4 collaboration, S. Agostinelli et al., GEANT4: a simulation toolkit, Nucl. Instrum. Meth. A 506 (2003) 250 [inSPIRE].

[40] M. Clemencic et al., The LHCb simulation application, GAUSs: design, evolution and experience, J. Phys. Conf. Ser. 331 (2011) 032023.

[41] D. Karlen, Using projections and correlations to approximate probability distributions, Comput. Phys. 12 (1998) 380 [physics/9805018] [INSPIRE]. 
[42] D. Martínez Santos, Study of the very rare decay $B_{s}^{0} \rightarrow \mu^{+} \mu^{-}$in LHCb , Ph.D. thesis, Universidade de Santiago de Compostela, Santiago de Compostela, Spain (2010), CERN-THESIS-2010-068.

[43] T. Skwarnicki, A study of the radiative cascade transitions between the $\Upsilon^{\prime}$ and $\Upsilon$ resonances, Ph.D. thesis, Institute of Nuclear Physics, Krakow, Poland (1986), DESY-F31-86-02.

[44] ARGUS collaboration, H. Albrecht et al., Exclusive hadronic decays of B mesons, Z. Phys. C 48 (1990) 543 [INSPIRE].

[45] D. Aston et al., A study of $K^{-} \pi^{+}$scattering in the reaction $K^{-} p \rightarrow K^{-} \pi^{+} n$ at $11 \mathrm{GeV} / c$, Nucl. Phys. B 296 (1988) 493 [INSPIRE].

[46] LHCb collaboration, Determination of $f_{s} / f_{d}$ for $7 \mathrm{TeV} p p$ collisions and a measurement of the $B^{0} \rightarrow D^{-} K^{+}$branching fraction, Phys. Rev. Lett. 107 (2011) 211801 [arXiv:1106.4435] [INSPIRE].

[47] A.S. Dighe, I. Dunietz, H.J. Lipkin and J.L. Rosner, Angular distributions and lifetime differences in $B_{s} \rightarrow J / \psi \phi$ decays, Phys. Lett. B 369 (1996) 144 [hep-ph/9511363] [InSPIRE]. 


\section{The LHCb collaboration}

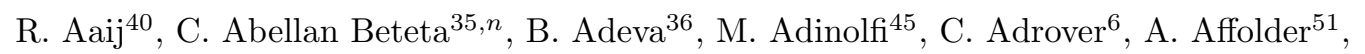
Z. Ajaltouni ${ }^{5}$, J. Albrecht ${ }^{9}$, F. Alessio ${ }^{37}$, M. Alexander ${ }^{50}$, S. Ali ${ }^{40}$, G. Alkhazov ${ }^{29}$, P. Alvarez Cartelle ${ }^{36}$, A.A. Alves $\mathrm{Jr}^{24,37}$, S. Amato ${ }^{2}$, S. Amerio ${ }^{21}$, Y. Amhis ${ }^{7}$, L. Anderlini ${ }^{17, f}$, J. Anderson ${ }^{39}$, R. Andreassen ${ }^{56}$, R.B. Appleby ${ }^{53}$, O. Aquines Gutierrez ${ }^{10}$, F. Archilli ${ }^{18}$, A. Artamonov ${ }^{34}$, M. Artuso 57 , E. Aslanides ${ }^{6}$, G. Auriemma ${ }^{24, m}$, S. Bachmann ${ }^{11}$, J.J. Back ${ }^{47}$, C. Baesso ${ }^{58}$, V. Balagura ${ }^{30}$, W. Baldini ${ }^{16}$, R.J. Barlow ${ }^{53}$, C. Barschel ${ }^{37}$, S. Barsuk ${ }^{7}$, W. Barter ${ }^{46}$, Th. Bauer ${ }^{40}$, A. Bay ${ }^{38}$, J. Beddow ${ }^{50}$, F. Bedeschi ${ }^{22}$, I. Bediaga ${ }^{1}$, S. Belogurov ${ }^{30}$, K. Belous ${ }^{34}$, I. Belyaev ${ }^{30}$, E. Ben-Haim ${ }^{8}$, M. Benayoun ${ }^{8}$, G. Bencivenni ${ }^{18}$, S. Benson ${ }^{49}$, J. Benton ${ }^{45}$, A. Berezhnoy ${ }^{31}$, R. Bernet ${ }^{39}$, M.-O. Bettler ${ }^{46}$, M. van Beuzekom ${ }^{40}$, A. Bien ${ }^{11}$, S. Bifani ${ }^{44}$, T. Bird ${ }^{53}$, A. Bizzeti ${ }^{17, h}$, P.M. Bjørnstad ${ }^{53}$, T. Blake ${ }^{37}$, F. Blanc ${ }^{38}$, J. Blouw ${ }^{11}$, S. Blusk ${ }^{57}$, V. Bocci ${ }^{24}$, A. Bondar ${ }^{33}$, N. Bondar ${ }^{29}$, W. Bonivento ${ }^{15}$, S. Borghi ${ }^{53}$, A. Borgia ${ }^{57}$, T.J.V. Bowcock ${ }^{51}$, E. Bowen ${ }^{39}$, C. Bozzi ${ }^{16}$, T. Brambach ${ }^{9}$, J. van den Brand ${ }^{41}$, J. Bressieux ${ }^{38}$, D. Brett ${ }^{53}$, M. Britsch ${ }^{10}$, T. Britton ${ }^{57}$, N.H. Brook ${ }^{45}$, H. Brown ${ }^{51}$, I. Burducea ${ }^{28}$, A. Bursche ${ }^{39}$, G. Busetto ${ }^{21, p}$, J. Buytaert ${ }^{37}$, S. Cadeddu ${ }^{15}$, O. Callot ${ }^{7}$, M. Calvi ${ }^{20, j}$, M. Calvo Gomez ${ }^{35, n}$, A. Camboni ${ }^{35}$, P. Campana ${ }^{18,37}$, D. Campora Perez ${ }^{37}$, A. Carbone ${ }^{14, c}$, G. Carboni ${ }^{23, k}$, R. Cardinale ${ }^{19, i}$, A. Cardini ${ }^{15}$, H. Carranza-Mejia ${ }^{49}$, L. Carson ${ }^{52}$, K. Carvalho Akiba ${ }^{2}$, G. Casse ${ }^{51}$, L. Castillo Garcia ${ }^{37}$, M. Cattaneo ${ }^{37}$, Ch. Cauet ${ }^{9}$, M. Charles ${ }^{54}$, Ph. Charpentier ${ }^{37}$, P. Chen ${ }^{3,38}$, N. Chiapolini ${ }^{39}$, M. Chrzaszcz ${ }^{25}$, K. Ciba ${ }^{37}$, X. Cid Vidal ${ }^{37}$, G. Ciezarek $^{52}$, P.E.L. Clarke ${ }^{49}$, M. Clemencic ${ }^{37}$, H.V. Cliff ${ }^{46}$, J. Closier ${ }^{37}$, C. Coca ${ }^{28}$, V. Coco ${ }^{40}$, J. Cogan ${ }^{6}$, E. Cogneras ${ }^{5}$, P. Collins ${ }^{37}$, A. Comerma-Montells ${ }^{35}$, A. Contu ${ }^{15}$, A. Cook ${ }^{45}$, M. Coombes ${ }^{45}$, S. Coquereau ${ }^{8}$, G. Corti ${ }^{37}$, B. Couturier ${ }^{37}$, G.A. Cowan ${ }^{49}$, D.C. Craik ${ }^{47}$, S. Cunliffe ${ }^{52}$, R. Currie $^{49}$, C. D’Ambrosio ${ }^{37}$, P. David ${ }^{8}$, P.N.Y. David ${ }^{40}$, A. Davis ${ }^{56}$, I. De Bonis ${ }^{4}$, K. De Bruyn ${ }^{40}$, S. De Capua ${ }^{53}$, M. De Cian $^{39}$, J.M. De Miranda ${ }^{1}$, L. De Paula ${ }^{2}$, W. De Silva ${ }^{56}$, P. De Simone ${ }^{18}$, D. Decamp ${ }^{4}$, M. Deckenhoff ${ }^{9}$, L. Del Buono ${ }^{8}$, D. Derkach ${ }^{14}$, O. Deschamps ${ }^{5}$, F. Dettori ${ }^{41}$, A. Di Canto ${ }^{11}$, H. Dijkstra ${ }^{37}$, M. Dogaru ${ }^{28}$, S. Donleavy $^{51}$, F. Dordei $^{11}$,

A. Dosil Suárez ${ }^{36}$, D. Dossett ${ }^{47}$, A. Dovbnya ${ }^{42}$, F. Dupertuis ${ }^{38}$, R. Dzhelyadin ${ }^{34}$, A. Dziurda ${ }^{25}$, A. Dzyuba ${ }^{29}$, S. Easo ${ }^{48,37}$, U. Egede ${ }^{52}$, V. Egorychev ${ }^{30}$, S. Eidelman ${ }^{33}$, D. van Eijk ${ }^{40}$, S. Eisenhardt ${ }^{49}$, U. Eitschberger ${ }^{9}$, R. Ekelhof ${ }^{9}$, L. Eklund ${ }^{50,37}$, I. El Rifai ${ }^{5}$, Ch. Elsasser ${ }^{39}$, D. Elsby ${ }^{44}$, A. Falabella ${ }^{14, e}$, C. Färber ${ }^{11}$, G. Fardell ${ }^{49}$, C. Farinelli ${ }^{40}$, S. Farry ${ }^{12}$, V. Fave ${ }^{38}$, D. Ferguson ${ }^{49}$, V. Fernandez Albor ${ }^{36}$, F. Ferreira Rodrigues ${ }^{1}$, M. Ferro-Luzzi ${ }^{37}$, S. Filippov ${ }^{32}$, M. Fiore ${ }^{16}$, C. Fitzpatrick ${ }^{37}$, M. Fontana ${ }^{10}$, F. Fontanelli ${ }^{19, i}$, R. Forty ${ }^{37}$, O. Francisco ${ }^{2}$, M. Frank ${ }^{37}$, C. Frei ${ }^{37}$, M. Frosini ${ }^{17, f}$, S. Furcas ${ }^{20}$, E. Furfaro ${ }^{23, k}$, A. Gallas Torreira ${ }^{36}$, D. Galli ${ }^{14, c}$, M. Gandelman ${ }^{2}$, P. Gandini ${ }^{57}$, Y. Gao ${ }^{3}$, J. Garofoli ${ }^{57}$, P. Garosi ${ }^{53}$, J. Garra Tico ${ }^{46}$, L. Garrido ${ }^{35}$, C. Gaspar ${ }^{37}$, R. Gauld ${ }^{54}$, E. Gersabeck ${ }^{11}$, M. Gersabeck ${ }^{53}$, T. Gershon ${ }^{47,37}$, Ph. Ghez ${ }^{4}$, V. Gibson ${ }^{46}$, V.V. Gligorov ${ }^{37}$, C. Göbel ${ }^{58}$, D. Golubkov ${ }^{30}$, A. Golutvin ${ }^{52,30,37}$, A. Gomes ${ }^{2}$, H. Gordon ${ }^{54}$, M. Grabalosa Gándara ${ }^{5}$, R. Graciani Diaz ${ }^{35}$, L.A. Granado Cardoso ${ }^{37}$, E. Graugés ${ }^{35}$, G. Graziani ${ }^{17}$, A. Grecu ${ }^{28}$, E. Greening ${ }^{54}$, S. Gregson ${ }^{46}$, O. Grünberg ${ }^{59}$, B. Gui ${ }^{57}$, E. Gushchin ${ }^{32}$, Yu. Guz ${ }^{34,37}$, T. Gys ${ }^{37}$, C. Hadjivasiliou ${ }^{57}$, G. Haefeli ${ }^{38}$, C. Haen ${ }^{37}$, S.C. Haines ${ }^{46}$, S. Hall ${ }^{52}$, T. Hampson ${ }^{45}$, S. Hansmann-Menzemer ${ }^{11}$, N. Harnew ${ }^{54}$, S.T. Harnew ${ }^{45}$, J. Harrison ${ }^{53}$, T. Hartmann ${ }^{59}$, J. He ${ }^{37}$, V. Heijne ${ }^{40}$, K. Hennessy ${ }^{51}$, P. Henrard ${ }^{5}$, J.A. Hernando Morata ${ }^{36}$, E. van Herwijnen ${ }^{37}$, A. Hicheur ${ }^{1}$, E. Hicks ${ }^{51}$, D. Hill ${ }^{54}$, M. Hoballah ${ }^{5}$, M. Holtrop ${ }^{40}$, C. Hombach ${ }^{53}$, P. Hopchev ${ }^{4}$, W. Hulsbergen ${ }^{40}$, P. Hunt ${ }^{54}$, T. Huse ${ }^{51}$, N. Hussain ${ }^{54}$, D. Hutchcroft ${ }^{51}$, D. Hynds ${ }^{50}$, V. Iakovenko ${ }^{43}$, M. Idzik ${ }^{26}$, P. Ilten ${ }^{12}$, R. Jacobsson ${ }^{37}$, A. Jaeger ${ }^{11}$, E. Jans ${ }^{40}$, P. Jaton ${ }^{38}$, F. Jing ${ }^{3}$, M. John ${ }^{54}$, D. Johnson ${ }^{54}$, C.R. Jones ${ }^{46}$, C. Joram ${ }^{37}$, B. Jost ${ }^{37}$, M. Kaballo ${ }^{9}$, S. Kandybei ${ }^{42}$, M. Karacson ${ }^{37}$, T.M. Karbach ${ }^{37}$, I.R. Kenyon ${ }^{44}$, U. Kerzel ${ }^{37}$,

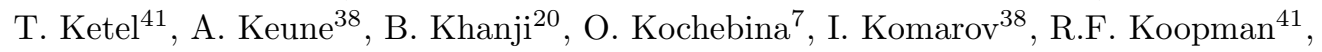


P. Koppenburg ${ }^{40}$, M. Korolev ${ }^{31}$, A. Kozlinskiy ${ }^{40}$, L. Kravchuk ${ }^{32}$, K. Kreplin ${ }^{11}$, M. Kreps ${ }^{47}$, G. Krocker ${ }^{11}$, P. Krokovny ${ }^{33}$, F. Kruse ${ }^{9}$, M. Kucharczyk ${ }^{20,25, j}$, V. Kudryavtsev ${ }^{33}$,

T. Kvaratskheliya ${ }^{30,37}$, V.N. La Thi ${ }^{38}$, D. Lacarrere ${ }^{37}$, G. Lafferty ${ }^{53}$, A. Lai ${ }^{15}$, D. Lambert ${ }^{49}$, R.W. Lambert ${ }^{41}$, E. Lanciotti ${ }^{37}$, G. Lanfranchi ${ }^{18,37}$, C. Langenbruch ${ }^{37}$, T. Latham ${ }^{47}$,

C. Lazzeroni ${ }^{44}$, R. Le Gac ${ }^{6}$, J. van Leerdam ${ }^{40}$, J.-P. Lees ${ }^{4}$, R. Lefèvre ${ }^{5}$, A. Leflat ${ }^{31}$, J. Lefrançois ${ }^{7}$,

S. Leo ${ }^{22}$, O. Leroy ${ }^{6}$, T. Lesiak ${ }^{25}$, B. Leverington ${ }^{11}$, Y. Li ${ }^{3}$, L. Li Gioi ${ }^{5}$, M. Liles ${ }^{51}$, R. Lindner ${ }^{37}$,

C. Linn ${ }^{11}$, B. Liu ${ }^{3}$, G. Liu ${ }^{37}$, S. Lohn ${ }^{37}$, I. Longstaff ${ }^{50}$, J.H. Lopes ${ }^{2}$, E. Lopez Asamar ${ }^{35}$,

N. Lopez-March ${ }^{38}, \mathrm{H} . \mathrm{Lu}^{3}$, D. Lucchesi ${ }^{21, p}$, J. Luisier ${ }^{38}, \mathrm{H}_{\text {. Luo }}^{49}$, F. Machefert ${ }^{7}$,

I.V. Machikhiliyan ${ }^{4,30}$, F. Maciuc ${ }^{28}$, O. $\mathrm{Maev}^{29,37}$, S. Malde ${ }^{54}$, G. Manca ${ }^{15, d}$, G. Mancinelli ${ }^{6}$,

U. Marconi ${ }^{14}$, R. Märki ${ }^{38}$, J. Marks ${ }^{11}$, G. Martellotti ${ }^{24}$, A. Martens ${ }^{8}$, A. Martín Sánchez ${ }^{7}$,

M. Martinelli ${ }^{40}$, D. Martinez Santos ${ }^{41}$, D. Martins Tostes ${ }^{2}$, A. Massafferri ${ }^{1}$, R. Matev ${ }^{37}$,

Z. Mathe ${ }^{37}$, C. Matteuzzi ${ }^{20}$, E. Maurice ${ }^{6}$, A. Mazurov ${ }^{16,32,37, e}$, J. McCarthy ${ }^{44}$, A. McNab ${ }^{53}$,

R. McNulty ${ }^{12}$, B. Meadows ${ }^{56,54}$, F. Meier ${ }^{9}$, M. Meissner ${ }^{11}$, M. Merk ${ }^{40}$, D.A. Milanes ${ }^{8}$,

M.-N. Minard ${ }^{4}$, J. Molina Rodriguez ${ }^{58}$, S. Monteil ${ }^{5}$, D. Moran ${ }^{53}$, P. Morawski ${ }^{25}$, M.J. Morello ${ }^{22, r}$,

R. Mountain ${ }^{57}$, I. Mous ${ }^{40}$, F. Muheim ${ }^{49}$, K. Müller ${ }^{39}$, R. Muresan ${ }^{28}$, B. Muryn ${ }^{26}$, B. Muster ${ }^{38}$,

P. Naik ${ }^{45}$, T. Nakada ${ }^{38}$, R. Nandakumar ${ }^{48}$, I. Nasteva ${ }^{1}$, M. Needham ${ }^{49}$, N. Neufeld ${ }^{37}$,

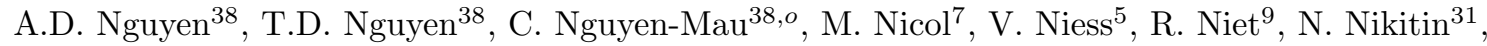

T. Nikodem ${ }^{11}$, A. Nomerotski ${ }^{54}$, A. Novoselov ${ }^{34}$, A. Oblakowska-Mucha ${ }^{26}$, V. Obraztsov ${ }^{34}$,

S. Oggero ${ }^{40}$, S. Ogilvy ${ }^{50}$, O. Okhrimenko ${ }^{43}$, R. Oldeman ${ }^{15, d}$, M. Orlandea ${ }^{28}$,

J.M. Otalora Goicochea ${ }^{2}$, P. Owen ${ }^{52}$, A. Oyanguren ${ }^{35}$, B.K. Pal ${ }^{57}$, A. Palano ${ }^{13, b}$, M. Palutan ${ }^{18}$,

J. Panman ${ }^{37}$, A. Papanestis ${ }^{48}$, M. Pappagallo ${ }^{50}$, C. Parkes $^{53}$, C.J. Parkinson ${ }^{52}$, G. Passaleva ${ }^{17}$,

G.D. Patel ${ }^{51}$, M. Patel ${ }^{52}$, G.N. Patrick ${ }^{48}$, C. Patrignani ${ }^{19, i}$, C. Pavel-Nicorescu ${ }^{28}$,

A. Pazos Alvarez ${ }^{36}$, A. Pellegrino ${ }^{40}$, G. Penso ${ }^{24, l}$, M. Pepe Altarelli ${ }^{37}$, S. Perazzini ${ }^{14, c}$,

D.L. Perego ${ }^{20, j}$, E. Perez Trigo ${ }^{36}$, A. Pérez-Calero Yzquierdo ${ }^{35}$, P. Perret ${ }^{5}$, M. Perrin-Terrinn ${ }^{6}$,

G. Pessina ${ }^{20}$, K. Petridis ${ }^{52}$, A. Petrolini ${ }^{19, i}$, A. Phan ${ }^{57}$, E. Picatoste Olloqui ${ }^{35}$, B. Pietrzyk ${ }^{4}$,

T. Pilařr $\check{r}^{47}$, D. Pinci ${ }^{24}$, S. Playfer ${ }^{49}$, M. Plo Casasus ${ }^{36}$, F. Polci ${ }^{8}$, G. Polok ${ }^{25}$, A. Poluektov ${ }^{47,33}$,

E. Polycarpo ${ }^{2}$, D. Popov ${ }^{10}$, B. Popovici ${ }^{28}$, C. Potterat ${ }^{35}$, A. Powell ${ }^{54}$, J. Prisciandaro ${ }^{38}$,

A. Pritchard ${ }^{51}$, C. Prouve ${ }^{7}$, V. Pugatch ${ }^{43}$, A. Puig Navarro ${ }^{38}$, G. Punzi ${ }^{22, q}$, W. Qian ${ }^{4}$,

J.H. Rademacker ${ }^{45}$, B. Rakotomiaramanana ${ }^{38}$, M.S. Rangel ${ }^{2}$, I. Raniuk ${ }^{42}$, N. Rauschmayr ${ }^{37}$,

G. Raven ${ }^{41}$, S. Redford ${ }^{54}$, M.M. Reid ${ }^{47}$, A.C. dos Reis ${ }^{1}$, S. Ricciardi ${ }^{48}$, A. Richards ${ }^{52}$,

K. Rinnert ${ }^{51}$, V. Rives Molina ${ }^{35}$, D.A. Roa Romero ${ }^{5}$, P. Robbe $^{7}$, E. Rodrigues ${ }^{53}$,

P. Rodriguez Perez ${ }^{36}$, S. Roiser ${ }^{37}$, V. Romanovsky ${ }^{34}$, A. Romero Vidal ${ }^{36}$, J. Rouvinet ${ }^{38}$, T. Ruf ${ }^{37}$,

F. Ruffini ${ }^{22}$, H. Ruiz ${ }^{35}$, P. Ruiz Valls ${ }^{35}$, G. Sabatino ${ }^{24, k}$, J.J. Saborido Silva ${ }^{36}$, N. Sagidova ${ }^{29}$,

P. Sail ${ }^{50}$, B. Saitta ${ }^{15, d}$, C. Salzmann ${ }^{39}$, B. Sanmartin Sedes ${ }^{36}$, M. Sannino ${ }^{19, i}$, R. Santacesaria ${ }^{24}$,

C. Santamarina Rios ${ }^{36}$, E. Santovetti ${ }^{23, k}$, M. Sapunov ${ }^{6}$, A. Sarti ${ }^{18, l}$, C. Satriano ${ }^{24, m}$, A. Satta ${ }^{23}$,

M. Savrie ${ }^{16, e}$, D. Savrina ${ }^{30,31}$, P. Schaack ${ }^{52}$, M. Schiller ${ }^{41}$, H. Schindler ${ }^{37}$, M. Schlupp ${ }^{9}$,

M. Schmelling ${ }^{10}$, B. Schmidt ${ }^{37}$, O. Schneider ${ }^{38}$, A. Schopper ${ }^{37}$, M.-H. Schune ${ }^{7}$, R. Schwemmer ${ }^{37}$,

B. Sciascia ${ }^{18}$, A. Sciubba ${ }^{24}$, M. Seco ${ }^{36}$, A. Semennikov ${ }^{30}$, I. Sepp ${ }^{52}$, N. Serra ${ }^{39}$, J. Serrano ${ }^{6}$,

P. Seyfert ${ }^{11}$, M. Shapkin ${ }^{34}$, I. Shapoval ${ }^{16,42}$, P. Shatalov ${ }^{30}$, Y. Shcheglov ${ }^{29}$, T. Shears ${ }^{51,37}$,

L. Shekhtman ${ }^{33}$, O. Shevchenko ${ }^{42}$, V. Shevchenko ${ }^{30}$, A. Shires ${ }^{52}$, R. Silva Coutinho ${ }^{47}$,

T. Skwarnicki ${ }^{57}$, N.A. Smith ${ }^{51}$, E. Smith ${ }^{54,48}$, M. Smith ${ }^{53}$, M.D. Sokoloff ${ }^{56}$, F.J.P. Soler ${ }^{50}$,

F. Soomro ${ }^{18}$, D. Souza ${ }^{45}$, B. Souza De Paula ${ }^{2}$, B. Spaan ${ }^{9}$, A. Sparkes ${ }^{49}$, P. Spradlin ${ }^{50}$,

F. Stagni ${ }^{37}$, S. Stahl ${ }^{11}$, O. Steinkamp ${ }^{39}$, S. Stoica ${ }^{28}$, S. Stone ${ }^{57}$, B. Storaci ${ }^{39}$, M. Straticiuc ${ }^{28}$,

U. Straumann ${ }^{39}$, V.K. Subbiah ${ }^{37}$, S. Swientek ${ }^{9}$, V. Syropoulos ${ }^{41}$, M. Szczekowski ${ }^{27}$,

P. Szczypka ${ }^{38,37}$, T. Szumlak ${ }^{26}$, S. T'Jampens ${ }^{4}$, M. Teklishyn ${ }^{7}$, E. Teodorescu ${ }^{28}$, F. Teubert ${ }^{37}$,

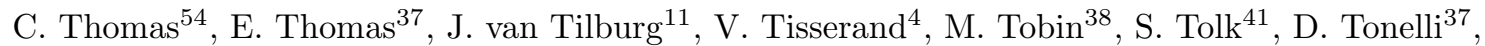

S. Topp-Joergensen ${ }^{54}$, N. Torr ${ }^{54}$, E. Tournefier ${ }^{4,52}$, S. Tourneur ${ }^{38}$, M.T. Tran ${ }^{38}$, M. Tresch ${ }^{39}$,

A. Tsaregorodtsev ${ }^{6}$, P. Tsopelas ${ }^{40}$, N. Tuning ${ }^{40}$, M. Ubeda Garcia ${ }^{37}$, A. Ukleja ${ }^{27}$, D. Urner ${ }^{53}$, 
U. Uwer ${ }^{11}$, V. Vagnoni ${ }^{14}$, G. Valenti ${ }^{14}$, R. Vazquez Gomez ${ }^{35}$, P. Vazquez Regueiro ${ }^{36}$, S. Vecchi ${ }^{16}$, J.J. Velthuis ${ }^{45}$, M. Veltri ${ }^{17, g}$, G. Veneziano ${ }^{38}$, M. Vesterinen ${ }^{37}$, B. Viaud ${ }^{7}$, D. Vieira ${ }^{2}$, X. Vilasis-Cardona ${ }^{35, n}$, A. Vollhardt ${ }^{39}$, D. Volyanskyy ${ }^{10}$, D. Voong ${ }^{45}$, A. Vorobyev ${ }^{29}$, V. Vorobyev ${ }^{33}$, C. Voß ${ }^{59}$, H. Voss ${ }^{10}$, R. Waldi ${ }^{59}$, R. Wallace ${ }^{12}$, S. Wandernoth ${ }^{11}$, J. Wang ${ }^{57}$, D.R. Ward ${ }^{46}$, N.K. Watson ${ }^{44}$, A.D. Webber ${ }^{53}$, D. Websdale ${ }^{52}$, M. Whitehead ${ }^{47}$, J. Wicht ${ }^{37}$, J. Wiechczynski ${ }^{25}$, D. Wiedner ${ }^{11}$, L. Wiggers ${ }^{40}$, G. Wilkinson ${ }^{54}$, M.P. Williams ${ }^{47,48}$, M. Williams ${ }^{55}$, F.F. Wilson ${ }^{48}$, J. Wishahi ${ }^{9}$, M. Witek ${ }^{25}$, S.A. Wotton ${ }^{46}$, S. Wright ${ }^{46}$, S. Wu ${ }^{3}$, K. Wyllie ${ }^{37}$, Y. Xie ${ }^{49,37}$, Z. Xing ${ }^{57}$, Z. Yang ${ }^{3}$, R. Young ${ }^{49}$, X. Yuan ${ }^{3}$, O. Yushchenko ${ }^{34}$, M. Zangoli ${ }^{14}$, M. Zavertyaev ${ }^{10, a}$, F. Zhang ${ }^{3}$, L. Zhang ${ }^{57}$, W.C. Zhang ${ }^{12}$, Y. Zhang ${ }^{3}$, A. Zhelezov ${ }^{11}$, A. Zhokhov ${ }^{30}$, L. Zhong ${ }^{3}$, A. Zvyagin ${ }^{37}$

1 Centro Brasileiro de Pesquisas Físicas (CBPF), Rio de Janeiro, Brazil

2 Universidade Federal do Rio de Janeiro (UFRJ), Rio de Janeiro, Brazil

3 Center for High Energy Physics, Tsinghua University, Beijing, China

4 LAPP, Université de Savoie, CNRS/IN2P3, Annecy-Le-Vieux, France

5 Clermont Université, Université Blaise Pascal, CNRS/IN2P3, LPC, Clermont-Ferrand, France

6 CPPM, Aix-Marseille Université, CNRS/IN2P3, Marseille, France

7 LAL, Université Paris-Sud, CNRS/IN2P3, Orsay, France

8 LPNHE, Université Pierre et Marie Curie, Université Paris Diderot, CNRS/IN2P3, Paris, France

9 Fakultät Physik, Technische Universität Dortmund, Dortmund, Germany

10 Max-Planck-Institut für Kernphysik (MPIK), Heidelberg, Germany

11 Physikalisches Institut, Ruprecht-Karls-Universität Heidelberg, Heidelberg, Germany

12 School of Physics, University College Dublin, Dublin, Ireland

13 Sezione INFN di Bari, Bari, Italy

14 Sezione INFN di Bologna, Bologna, Italy

15 Sezione INFN di Cagliari, Cagliari, Italy

16 Sezione INFN di Ferrara, Ferrara, Italy

17 Sezione INFN di Firenze, Firenze, Italy

18 Laboratori Nazionali dell'INFN di Frascati, Frascati, Italy

19 Sezione INFN di Genova, Genova, Italy

20 Sezione INFN di Milano Bicocca, Milano, Italy

21 Sezione INFN di Padova, Padova, Italy

22 Sezione INFN di Pisa, Pisa, Italy

23 Sezione INFN di Roma Tor Vergata, Roma, Italy

24 Sezione INFN di Roma La Sapienza, Roma, Italy

${ }^{25}$ Henryk Niewodniczanski Institute of Nuclear Physics Polish Academy of Sciences, Kraków, Poland

26 AGH - University of Science and Technology, Faculty of Physics and Applied Computer Science, Kraków, Poland

27 National Center for Nuclear Research (NCBJ), Warsaw, Poland

28 Horia Hulubei National Institute of Physics and Nuclear Engineering, Bucharest-Magurele, Romania

29 Petersburg Nuclear Physics Institute (PNPI), Gatchina, Russia

30 Institute of Theoretical and Experimental Physics (ITEP), Moscow, Russia

31 Institute of Nuclear Physics, Moscow State University (SINP MSU), Moscow, Russia

32 Institute for Nuclear Research of the Russian Academy of Sciences (INR RAN), Moscow, Russia

33 Budker Institute of Nuclear Physics (SB RAS) and Novosibirsk State University, Novosibirsk, Russia

34 Institute for High Energy Physics (IHEP), Protvino, Russia

35 Universitat de Barcelona, Barcelona, Spain

36 Universidad de Santiago de Compostela, Santiago de Compostela, Spain

37 European Organization for Nuclear Research (CERN), Geneva, Switzerland

38 Ecole Polytechnique Fédérale de Lausanne (EPFL), Lausanne, Switzerland 
Physik-Institut, Universität Zürich, Zürich, Switzerland

40 Nikhef National Institute for Subatomic Physics, Amsterdam, The Netherlands

41 Nikhef National Institute for Subatomic Physics and VU University Amsterdam, Amsterdam, The Netherlands

42 NSC Kharkiv Institute of Physics and Technology (NSC KIPT), Kharkiv, Ukraine

43 Institute for Nuclear Research of the National Academy of Sciences (KINR), Kyiv, Ukraine

44 University of Birmingham, Birmingham, United Kingdom

45 H.H. Wills Physics Laboratory, University of Bristol, Bristol, United Kingdom

46 Cavendish Laboratory, University of Cambridge, Cambridge, United Kingdom

47 Department of Physics, University of Warwick, Coventry, United Kingdom

48 STFC Rutherford Appleton Laboratory, Didcot, United Kingdom

49 School of Physics and Astronomy, University of Edinburgh, Edinburgh, United Kingdom

50 School of Physics and Astronomy, University of Glasgow, Glasgow, United Kingdom

51 Oliver Lodge Laboratory, University of Liverpool, Liverpool, United Kingdom

52 Imperial College London, London, United Kingdom

53 School of Physics and Astronomy, University of Manchester, Manchester, United Kingdom

54 Department of Physics, University of Oxford, Oxford, United Kingdom

55 Massachusetts Institute of Technology, Cambridge, MA, United States

56 University of Cincinnati, Cincinnati, OH, United States

57 Syracuse University, Syracuse, NY, United States

58 Pontifícia Universidade Católica do Rio de Janeiro (PUC-Rio), Rio de Janeiro, Brazil, associated $t^{2}$

59 Institut für Physik, Universität Rostock, Rostock, Germany, associated to ${ }^{11}$

a P.N. Lebedev Physical Institute, Russian Academy of Science (LPI RAS), Moscow, Russia

b Università di Bari, Bari, Italy

c Università di Bologna, Bologna, Italy

d Università di Cagliari, Cagliari, Italy

e Università di Ferrara, Ferrara, Italy

$f$ Università di Firenze, Firenze, Italy

$g$ Università di Urbino, Urbino, Italy

${ }^{h}$ Università di Modena e Reggio Emilia, Modena, Italy

i Università di Genova, Genova, Italy

j Università di Milano Bicocca, Milano, Italy

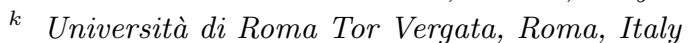

${ }^{l}$ Università di Roma La Sapienza, Roma, Italy

$m$ Università della Basilicata, Potenza, Italy

${ }^{n}$ LIFAELS, La Salle, Universitat Ramon Llull, Barcelona, Spain

o Hanoi University of Science, Hanoi, Viet Nam

$p$ Università di Padova, Padova, Italy

$q$ Università di Pisa, Pisa, Italy

${ }^{r}$ Scuola Normale Superiore, Pisa, Italy 